\title{
Designing of a mini-core that effectively represents 3004 diverse accessions of rice
} Angad Kumar $^{\# 1}$, Shivendra Kumar ${ }^{\# 1}$, Manoj Prasad ${ }^{1}$, Jitendra K. Thakur ${ }^{1 *}$

${ }^{1}$ National Institute of Plant Genome Research, Aruna Asaf Ali Marg, New Delhi 110067, India *Corresponding Author Email: jthakur@nipgr.ac.in

"Equal contribution

\section{One-sentence summary:}

Designing of mini-core as manageable association panel that efficiently mirroring the large and diverse collection of 3004 rice accessions.

Key words: Rice, mini-core, SNPs, GWAS, 3KRG, agronomic traits

\section{AUTHOR'S CONTRIBUTION}

A.K. performed all the analyses and contributed in writing; S.K. contributed in analyses and wrote the initial draft with contributions from all the authors; M.P. provided technical assistance; J.K.T. conceived the project, supervised and complemented the writing and agrees to serve as the author responsible for contact and ensures communication. 


\begin{abstract}
Genetic diversity provides foundation for plant breeding and genetic research. As part of the $3 \mathrm{~K}$ Rice Genome Project, over 3000 rice genomes were recently sequenced. We added four Indian rice accessions to it and made original panel of 3004 accessions. However, large set of germplasms are difficult to preserve and evaluate. Construction of core and mini-core collections is an efficient method for managing genetic resources. This study aims to designing of mini-core comprised of 520 accessions representing original panel. The designed mini-core captured most of the SNPs and represented all phenotypes and geographical regions. The mini-core was validated using different statistical analyses and had representation from all major groups including japonica, indica, aus/boro and aromatic/basmati. GWAS analyses with mini-core panel efficiently reproduced the marker-traits association identified among original 3004 panel. Expression analysis of trait-associated genes highlights the relevance of such mini-core panel. Haplotype analysis could also validate our mini-core panel. Apart from mini-core, we identified few regional and varietal specific marker-trait associations which were not evident in original panel. In this era of large-scale sequencing projects, such a strategy of designing mini-core will be very useful.
\end{abstract}

\title{
INTRODUCTION
}

Rice (Oryza sativa) is among the primary staple crop fulfilling the nutritional requirement of more than half of the world's population. An increase/improvement in its global production will have a direct impact on meeting the growing food demand of the world population. More than $90 \%$ of the world's rice production is contributed by Asian countries majorly dominated by China and India (FAOSTAT, 2017). India is the second largest producer of rice (165.3 million tons) after China (208.4 million tons) contributing $\sim 22 \%$ of the total rice production of the world. Increase in the rice yield is achieved mainly through improved cropping methods, use of fertilizers and in many areas by intense irrigation. However, outcome of these strategies is now reaching saturation and is becoming limited. So, there is demand of finding alternative ways for yield improvement. Genetic improvement of rice cultivars and varieties can be an effective mean in this regard. The goal can be realized through improved breeding programs using marker-assisted selection and genetics methods which will help in identification of new sources of genetic variations that may help in increasing the productivity (McCouch et al., 2016). 
Productivity/yield is a complex trait governed by multiple genes and depends upon the genetic composition and the environmental factors. The variability arises due to segregating alleles at multiple loci where the effect of each allele on the phenotypic trait is relatively small and the overall expression is also influenced by environmental conditions. Single nucleotide polymorphisms (SNPs) present throughout the genome are one of the major causes of allelic variation leading to genetic variability in a population. Quantitative genetic variations lead to a multitude of phenotypes which forms the basis for selection of improved cultivars for breeding and agricultural purpose. Identification of loci governing quantitative traits is critical for maintenance of variation within and among populations. Identification of quantitative trait loci (QTLs) by conventional method of linkage mapping or QTL mapping involves development of mapping population which is a time consuming process and captures limited number of recombination events based on the parental combinations. This methodology forms a part of markers assisted selection and biotechnological approach that has been utilized in large number of crops for identification of genes governing complex traits (Edgerton, 2009; Morrell et al., 2012).

However, with advancement in high-throughput genome sequencing and phenotyping methods, genome wide association studies (GWAS) have been initiated which have been proven to be more effective for crop improvement. It is a more efficient approach for identification of marker-trait association and has been utilized for recognition of genes or loci governing the complex trait (Singh and Singh, 2015; Huang et al., 2012; Morrell et al., 2012; Kump et al., 2011; Famoso et al., 2011; Ingvarsson and Street, 2011; Zhao et al., 2011; Huang et al., 2010; Breseghello and Sorrells, 2006; Gupta et al., 2005). The advantage of GWAS is that it does not require development of mapping population. It explores the naturally available population for mining of available genomic diversity to assess marker-trait association. It also captures large number of historical recombination events prevalent in the natural population. The basic requirement for GWAS is a diverse panel beholding historical recombination events for greater genetic resolution (Morrell et al., 2012). This purpose is best served by a core collection which is designed to capture maximum available/possible diversity (genetic, phenotypic and geographical) of the entire population, with limited number of individuals having low or no kinship among them (Korte et al., 2012). Core collections have been used as an association panel for GWAS in different studies (El Bakkali et al., 2013; Zhang et al., 2014; Perseguini et al., 2015; Ambreen et al., 2018). In case of rice, limited attempts have been made to use a core collection as an association panel. Recently, with the availability of resequencing dataset for 3024 diverse rice accessions, a further deep and robust platform has been provided to elevate the marker-associated breeding efforts concerning various agronomic traits (Li et al., 2014; Alexandrov et al., 2015; Mansueto et al., 2016). Follow up studies has also explored the detailed structural variation and introgression pattern among the $3 \mathrm{KRG}$ dataset, further strengthening our understanding for rice 
diverse genome and traits domestication (Wang et al., 2018) (Fuentes et al., 2019) . Even though this panel of 3000 accessions represents core collection of global rice accessions, but still its relatively large size would present difficulties for management and phenotype evaluation (Brown, 2011). So, the need to have a smaller subset mirroring such huge germplasm is warranted for convenient breeding efforts. Here, in this study, we have developed and utilized a mini-core collection (520 accessions) from the original collection of 3004 rice accession dataset, as an association panel for GWAS analysis using 2 million SNPs. While designing mini-core collection, we have considered genotypic (SNPs data), phenotypic data (18 yield-related traits), and representation from various regional genepools (geographical diversity) to accommodate maximum possible diversity. We demonstrate here that such analysis lead to identification of loci which can play an important role for increasing the yield of rice and will be helpful in identification of genes involved in regulation of these traits. The comparatively small size of the association panel designed in the study will be useful and convenient for various phenotype-genotype relationship studies which usually remain a major limitation in different plant breeding programs.

\section{RESULTS AND DISCUSSION}

\section{Genome sequencing and identification of sequence polymorphism}

We re-sequenced four rice accessions in our lab (LGR, PB 1121, Sonasal and Bindli) and identified 3564117 SNPs. We used the default parameters of BWA for alignment of reads sequences from all the four rice genotypes on Nipponbare reference genome. Only those SNPs with average base quality of 30 , minimum read depth of 10 and minimum polymorphism call rate of $>90 \%$ were scored in order to minimize the detection of false positives. All the SNPs which were consecutive and adjacent to InDels were also eliminated. The read depth of these SNPs varied from 10 to more than 11000, with the overall sequencing depth for four rice accessions ranging from 42 to $48 \mathrm{X}$. We clubbed these SNPs with the $3 \mathrm{~K}$ Rice Genome (3KRG) project SNP dataset for this study (Alexandrov et al., 2015). Overall 18.9 million single nucleotide polymorphisms were identified among the 3,000 sequenced genomes with an average depth of $\sim 14 \mathrm{X}$, ranging from $\sim 4 \mathrm{X}$ to $60 \mathrm{X}$. For our analysis, in order to bring the $3 \mathrm{KRG}$ dataset to same level of quality we considered the filtered dataset ( 48 lakhs SNPs), corrected with excess of heterozygosity and linkage disequilibrium (LD). Finally, we merged both the datasets and deduced the common SNPs ( 20 lakhs) between the two studies (3KRG and four Indian genotypes). The distribution of common SNPs over different rice chromosomes showed non-uniform distribution. Maximum number of SNPs were located on the chromosome 1, 11 and 2 while least number of SNPs were found to be present on chromosome 9. 


\section{Development of mini-core collection}

In order to make a representative mini-core group, we utilized phenotypic data of 2266 rice accessions available for 18 yield-related traits and genotypic data (SNPs) of 3000 accessions of 3KRG dataset. The purpose of developing independent mini-cores using phenotypic data and genotypic data was to avoid trade off and to capture maximum possible phenotypic and genotypic variability prevalent in the original collection of rice accessions. Scanning of 2266 accessions resulted into a mini-core collection (CC1) of 227 accessions representing 10\% of the initial collection. We added the four Indian accessions (LGR, PB 1121, Sonasal and Bindli) that were resequenced in our lab. Thus, the mini-core collection $\mathrm{CC} 1$ consisted of 231 accessions representing diversity in phenotypic traits. The 3000 accessions with their SNP data were analysed separately for development of another mini-core collection (CC2) consisting of 300 accessions representing $10 \%$ of the original collection and also included the four Indian accessions (LGR, PB 1121, Sonasal and Bindli) sequenced in our lab.

The mini-core collections, $\mathrm{CC} 1$ and $\mathrm{CC} 2$ were assessed for their coverage of phenotypic variations with reference to the original panel (Supplemental Table S1). It was found that none of these two mini-cores could capture the entire range for all the phenotypic traits prevalent in the original collection. The traits which could not be captured in the mini-cores included days to $80 \%$ heading, 100 grain weight, days to first flowering, grain width, panicle length and seedling height. These minicore collections ( $\mathrm{CC} 1$ and $\mathrm{CC} 2$ ) were further assessed for various evaluation criteria such as Shannon's diversity index $(I)$, Nei's gene diversity $(H)$, mean difference percentage (MD\%), variance difference percentage (VD\%), variable rate of coefficient of variance (VR\%) and coincidence rate of range $(\mathrm{CR} \%)$ to assess their efficiency to capture the maximum diversity prevalent in the original collection. The MD\% of the developed mini-core collections was in the range of $2.8-4.08 \%$ which was well below the prescribed value of $20 \%$ (Supplemental Table S2). VD\% representing the variance captured in the mini-core collections ranged from 19.78 to $39.77 \%$. The VR\% varied from 86 for CC1 to $107.68 \%$ for CC2. CC1 had the highest CR\% value of $92 \%$ whereas $\mathrm{CR} \%$ of $\mathrm{CC} 2$ was 91.1 . The value of Shannon-Weaver index $(H)$ ranged from 1.98 for $\mathrm{CC} 1$ to 2.25 for CC2. The value of Nei's genetic diversity $(I)$ was found to be higher for CC2 (0.79) in comparison to CC1 (0.77) (Supplemental Table S2).

The developed mini-core collections were also assessed for representation of all the varietal groups and regional genepools present in the original panel (Supplemental Table S3 \& S4). The mini-core CC1 had highest representation of indica (129) followed by Temperate japonica (38), Intermediate (19), Tropical japonica (15), japonica (14), aus/boro (11) and Aromatic (5). The mini-core CC2 had highest representation of indica (171) followed by Intermediate (45), aus/boro (42), Aromatic and japonica (12 each), Tropical japonica (10) and Temperate japonica (8) (Supplemental Table S3). We also assessed the comparative distribution of accessions from different varietal groups into the mini- 
cores and found that CC2 had higher proportion of accessions from aus/boro (19.5\% of the original representation), Intermediate (33.3\% of the original representation) and Aromatic (16.9\% of the original representation) groups. On the other hand, $\mathrm{CC} 1$ had only $5.1 \%$ representation of aus/boro, $14 \%$ of Intermediate and 7\% of Aromatic group from the original collection (Supplemental Table S3). Accessions from Temperate japonica group were highly represented in CC1 (11.9\% of the original representation) while only small proportion of Temperate japonica group (2.5\% of original representation) were picked in CC2 (Supplemental Table S3). Comparable portions of accessions from indica (7.4\% in $\mathrm{CC} 1$ and $9.8 \%$ in $\mathrm{CC} 2$ ), Tropical japonica (3.8\% in $\mathrm{CC} 1$ and $2.5 \%$ in $\mathrm{CC} 2$ ) and japonica (10.6\% in CC1 and 9\% in CC2) groups were represented in both CC1 and CC2 mini-cores. Thus, none of the two mini-cores developed here consisted of $10 \%$ of representations from all the varietal groups (Supplemental Table S3). The developed mini-core collections were also assessed for distribution of accessions from different regional genepools. The mini-core $\mathrm{CC} 1$ consisted of 55 accessions from South Asia (6.9\% of the original collection) followed by 52 accessions of South East Asia (5.1\% of the original collection), 52 accessions of China (10.8\% of original collection), 18 accessions of Europe (15.2\% of original collection), 17 accessions of America (10.2\% of original collection), 15 accessions each from East Asia and Africa (11.4\% and 5.9\% of the original collection, respectively), 4 accessions of Oceania (23.5\% of original collection) and 3 accessions of Unknown origin (8.8\% of original collection). CC2 comprised of 122 accessions from South Asia (15.5\% of the original collection) followed by 70 accessions of South East Asia (6.9\% of the original collection), 55 accessions of China (11.4\% of the original collection), 23 accessions of Africa (9.1\% of the original collection), 13 accessions of America (7.8\% of the original collection), 8 accessions of East Asia (6\% of the original collection), 6 accessions of Unknown origin (17.4\% of the original collection), 2 accessions of Europe (1\% of the original collection) and 1 accession from Oceania (5.9\% of the original collection; Supplemental Table S4).

Only 28 accessions were found to be common between CC1 and CC2 showing that different accessions were selected on the basis of phenotypic and genotypic variation and justifying our concern of designing the mini-cores independently using just phenotypic or genotypic data. An ideal mini-core should represent maximum possible diversity present in the original collection. However, different evaluation criteria such as phenotypic range (Supplemental Table S1), MD\%, VD\%, VR\%, CR\%, Shannon and Nei's diversity indices (Supplemental Table S2), varietal (Supplemental Table S3) and geographical coverage (Supplemental Table S4) revealed that none of the mini-cores (CC1 and $\mathrm{CC} 2$ ) were able to capture the complete diversity ranges of original collection to be considered as an ideal representative subset. Therefore, we merged $\mathrm{CC} 1$ and $\mathrm{CC} 2$ to develop mini-core collection (CC3) comprising of 520 non-redundant accessions (503 accessions by merging CC1 and CC2 + 17 accessions capturing the extreme values of phenotypic traits discussed below) in order to capture 
maximum possible allele/trait diversity and to prevent any trade-off between the two data sets (phenotypic and genotypic) when used in conjunction (Fig. 1). CC3 comprising of 520 accessions represented $17.3 \%$ of the initial collection (3004 accessions) fulfilling the initial requirement of size of an ideal core collection which should range between 5-20\% of the original collection (Brown and Spilllane, 1999). CC3 was assessed for its representation of the original collection and various traits under consideration by different evaluation criteria (Supplemental Table S1-4). CC3 covered the entire range of traits from original collection including the traits which were not completely covered by $\mathrm{CC} 1$ and $\mathrm{CC} 2$ such as days to $80 \%$ heading, 100 grain weight, days to first flowering, grain width, panicle length and seedling height (Supplemental Table S1). CC3 was evaluated by different evaluation criteria. The MD\% of $\mathrm{CC} 3$ was $2.9 \%$ within the range of $2.8-4.08 \%$ observed for $\mathrm{CC} 1$ and CC2 (Supplemental Table S2). The value of VD\% representing the variance captured by CC3 accessions was $18.9 \%$ which is lower than the value of $\mathrm{CC} 1$ and $\mathrm{CC} 2$. The value of VR\% captured in the mini-core CC3 was $109.3 \%$ (highest value among the three mini-cores). Also, CC3 was found to have the highest value of CR\% (96.2) among all the three mini-cores. The value of Shannon-Weaver index $(H)$ and Nei's genetic diversity $(I)$ for CC3 was found to be 2.17 and 0.79 , respectively (Supplemental Table S2).

The mini-core was also assessed for representation of all the varieties and regional gene pools of rice present in the initial collection (Supplemental Table S3 \& S4). In the mini-core CC3, all the varieties had at-least $10 \%$ representation from original collection except Tropical japonica varietal group which had only $6.9 \%$ representation of original collection (27 accessions; Supplemental Table S3). Number of accessions and percentage representation of all the varietal groups of the original collection in the mini-core CC3 has been provided in Supplemental Table S3. Representation of accessions from different regional genepools of original collection varied from 12 to $29.4 \%$ in the mini-core CC3 (Supplemental Table S4). CC3 had highest contribution from South Asia (176 accessions; $22.4 \%$ of its original representation $12 \%$ of its original representation), China (101 accessions; $20.95 \%$ of its original representation), Africa (38 accessions; $15 \%$ of its original representation), America (28 accessions; $16.9 \%$ of its original representation), East Asia (21 accessions; $15.9 \%$ of its original representation), Europe (19 accessions; $16.1 \%$ of its original representation), Unknown origin (9 accessions; $25.5 \%$ of its original representation) and Oceania (5 accessions; $29.4 \%$ of its original representation; Supplemental Table S4). Thus, mini-core collection CC3 with 520 accessions was found to fulfil all the criteria of capturing maximum possible diversity of the original panel (3004 accessions) in comparison to $\mathrm{CC} 1$ and $\mathrm{CC} 2$ developed using phenotypic and molecular data, respectively, and was considered further for its utility as an association panel. 


\section{Correlation analysis between quantitative traits}

Correlation analysis was performed between different quantitative traits under consideration. This exercise was done to identify the traits, if any, which were governed by same genes or which were developmentally or structurally related to each other. A significant positive correlation was observed between days to $80 \%$ heading (DEH) and days to first flower (DFF; 0.998). The correlation values for five trait combinations were found to be higher than 0.5 ; culm length and days to first flower $(0.68)$, culm diameter and days to first flower (0.614), culm length and panicle length (0.557), culm length and days to $80 \%$ heading (0.555) and grain width and 100 grain weight $(0.542)$. The correlation values for all the other trait combinations were found to be lower than 0.5 . Though some of the traits studied here found to be negatively correlated but the correlation was not significant. The correlation coefficient of all the traits analysed is provided in Supplemental Table S5. The magnitude and direction of correlation between different traits helps in the selection decisions in breeding and crop improvement programs.

\section{Distance-based cluster analysis and principal component analysis (PCA)}

Distance-based cluster analysis was performed to assess the grouping of accessions of original collection of rice genotypes. Analysis of the SNP data (2081521 SNPs) using Maximum-likelihood method grouped 3004 accessions into two major clusters (named as CL I and II) with internal subgroupings (Fig. 2A). The cluster CL I was found to contain maximum number of accessions (66\%) of original collection while CL II showed presence of around 32\% of accessions (Supplemental Table S6). Around $1.4 \%$ of the accessions did not belong to any of the groups (Fig. 2A). These un-clustered accessions (43) were mainly Intermediate (21) and indica (15) group genotypes. In addition, some of the japonica (3), tropical japonica (2), temperate japonica (1) and aromatic (1) genotypes also remained un-clustered. The cluster CL I comprise of 1987 accessions was further grouped into two sub-clusters CL Ia and CL Ib. The larger sub-cluster CL Ia consisting of 1771 accessions was majorly dominated by indica (1641) varieties whereas cluster CL Ib comprised of a total of 216 accessions with major contribution from aus/boro (172) followed by indica (25) varieties. CL II with 974 accessions was divided into three sub-clusters named as CL IIa, IIb and IIc. CL IIa being the largest sub-cluster of CCL II comprised of 519 accessions majorly dominated by Tropical japonica (329) and japonica (80). The sub-cluster CL Ilb consisting of 358 accessions was dominated by Temperate japonica (250) genotypes. The Indian genotype included in the study (LGR) was part of sub-cluster IIb. CL IIc was the smallest sub-cluster of CL II comprising of 97 accessions with major representation from Aromatic (50) and Intermediate (23) varieties. Bindli, Sonasal and PB 1121 grouped together in sub-cluster CL IIc (Fig. 2A; Supplemental Table S6).

In the Principal component analysis (PCA), 3004 accessions were evenly distributed on coordinate axes 1 and 2 covering $45.6 \%$ and $26 \%$ of total variance, respectively (Fig. 2B). Accessions from 
indica variety clustered together in PCA analysis showing congruence with distance- based analysis. Accessions from indica which forms the largest group of original collection and were majorly present in Cluster Ia of distance-based analysis were predominantly present in quadrant I and IV. Accessions from japonica, temperate japonica and tropical japonica which were part of Cluster II of distancebased analysis were found to be present in quadrant III and IV. Accessions from aus/boro were found to be limited in quadrant I while Aromatic accessions were found to be present in quadrant II. Accessions of Intermediate type were found to be spread in all the quadrants of PCA analysis and showed congruence with maximum likelihood dendrogram in which they were present among all the clusters in even proportion (Fig. 2B). Next, we checked the distribution of CC3 accessions on distance-based maximum likelihood dendrogram and PCA analysis of Initial collection of rice to assess their presence in all the clusters and quadrants. CC3 showed balanced representations from all the clusters in the range of 10.5\%-25.7\% of accessions of maximum likelihood dendrogram (Fig. 3A; Supplemental Table S7). A total of $18.1 \%$ of accessions from sub-cluster CL Ia, $19.4 \%$ of accessions from sub-cluster CL Ib, $25 \%$ of un-clustered accessions, $10.5 \%$ of accessions from sub-cluster IIa, $18 \%$ of accessions from sub-cluster IIb and $25.7 \%$ accessions from sub-cluster IIc were captured in CC3 from original collection (Fig. 3A; Supplemental Table S7). Similarly, accessions from all the quadrants of PCA analysis were found to be part of CC3 (Fig. 3B). Thus, CC3 had representation of accessions from all the clusters of maximum likelihood dendrogram and quadrants of PCA, capturing maximum possible genotypic diversity.

\section{Population structure analysis of original collection using FastSTRUCTURE}

Population structure analysis for initial collection of rice (3004 accessions) was performed using the FastSTRUCTURE program. The best clustering was at $\mathrm{K}=7$ and the clusters obtained were named as FSTR CL 1-7 (Fig. 4A). FSTR CL 1 consisted of 219 accessions majorly dominated by aus/boro (179) followed by indica (28; Supplemental Table S8) and showed congruence with CL Ib of maximum likelihood analysis. FSTR CL 2 comprised of 522 accessions having highest representation from tropical japonica (310) and japonica group (94) and was found to have similar accessions as in CL IIa of maximum likelihood analysis. Some of the indica (47 accessions), temperate japonica (35) and Intermediate group (27) accessions were also found in FSTR CL 2. The smallest cluster was FSTR CL 3 with 90 accessions and was dominated by Aromatic accessions (50) followed by Intermediate group accessions (19) and was showing congruence with CL IIc of maximum likelihood analysis. The largest cluster of population structure analysis was FSTR CL 4 comprising of 973 accessions with major representation from indica group (885) and minor contributions from Tropical japonica (26), Intermediate (21), Temperate japonica (17) and aus/boro group (14). FSTR CL 5 comprised of 372 accessions majorly representing Temperate japonica (248) and minor 
representations from Tropical japonica (35), Intermediate (29), indica (26) and japonica group (25) and was similar to CL IIb of maximum likelihood analysis. The FSTR CL 6 consisted of 323 accessions dominated by indica variety (297) while FSTR CL 7 comprised of a total of 505 accessions having contributions from indica (451) and Intermediate varieties (27). The FSTR CL 4, 6 and 7 corresponded with the CL Ia of maximum likelihood dendrogram, suggesting that the accessions of CLIa can be further divided into three subgroups. Number of accessions constituting different clusters as determined by FastSTRUCTURE analysis is provided in Supplemental Table S8. Next, we looked for admixed genotypes in all the groups and found that $41 \%$ (1242) of accessions were admixed in nature (Supplemental Table S9). Among all the clusters, FSTR CL 7 consisting of 505 accessions had more admixed individuals (351) than pure individuals (154 accessions) followed by FSTR CL 6 (145 admixed and 148 pure accessions; Supplemental Table S9). Assessment of admixtures within varietal groups revealed that Intermediate population had more admixtures (94) than pure (41) accessions while indica population had 821 admixed individuals (47\%) out of 1743 accessions (Supplemental Table S9). Analysis of regional genepools revealed that only European region had more admixed (65) than pure individuals (53) (Supplemental Table S9). Distribution of CC3 accessions (520) in different clusters of FastSTRUCTURE analysis (FSTR CL 1 - 7) was assessed to check representation of individuals from all the clusters in the developed mini-core collection. CC3 captured 50 accessions (40 pure individuals with Q value > 80\%) from a total of 219 accessions present in FSTR CL 1 (Supplemental Table S10). Forty two accessions (23 pure individual) were picked from 522 accessions of FSTR CL 2 in CC3 while 24 accessions (13 pure individuals) were part of CC3 from FSTR CL 3. CC3 captured 185 accessions (109 pure individuals) from the largest FastSTRUCTURE cluster FSTR CL 4 comprising of 973 accessions. Out of 372 accessions of FSTR CL 5, 74 accessions were present in CC3 (37 pure individuals). Out of the 323 accessions of FSTR CL 6, 61 accessions were part of CC3 (28 pure individuals) while 84 accessions (25 pure individuals) were present in CC3 representing FSTR CL 7 with 505 accessions (Supplemental Table S10). Thus, CC3 had representations of both pure and admixed accessions from all the 7 clusters of FastSTRUCTURE analysis derived for initial collection of rice. Therefore, we were able to verify the initial objective of development of a mini-core collection (CC3) representing maximum phenotypic, genotypic, varietal and geographical variability present in the original collection of rice consisting of 3004 rice accessions.

\section{Assessment of mini-core collection CC3 to assess its utility as an association panel}

An association panel should have low population structure and low kinship among its members to avoid spurious marker-trait associations (Yu and Buckler, 2006; Zhu et al., 2008; Yang et al., 2010; Nachimuthu et al., 2015). Therefore, we performed population structure and kinship analysis among the accessions of the developed mini-core collection (CC3) to use it as an association panel. 


\section{Population structure analysis of mini-core CC3}

Estimation of the underlying population structure among 520 accessions of mini-core collection CC3 was done using FastSTRUCTURE program which delineated them into seven clusters $(K=7)$ named as CC CL1-CC CL7 (Fig. 4B; Supplemental Table S11). The cluster 1 (CC CL 1) comprised of 73 accessions and was dominated by indica (57) and Intermediate group accessions (12). CC CL2 was the smallest of all the clusters comprising of 23 accessions dominated by Aromatic (9) and Intermediate group (8) genotypes. The cluster CC CL3 consisting of 49 accessions was dominated by aus/boro (41). CC CL4 comprised of 70 accessions and had representations from indica (58 accessions) and Intermediate (8) populations. The cluster CC CL5 consisted of 43 accessions belonging to different varietal groups such as Tropical japonica (17), japonica (10) and Intermediate group (8). CC CL6 was the largest cluster consisting of 191 accessions majorly belonging to indica (165) and Intermediate (11) populations. CC CL7 comprised of 77 accessions majorly representing Temperate japonica (36), Intermediate (14) and japonica (9) population. The detailed distribution of accessions belonging to different varietal groups in 7 clusters of mini-core collection $\mathrm{CC} 3$ has been provided in Supplemental Table S11.

We found that 47\% (245) of the accessions of mini-core collection CC3 were admixtures (Supplemental Table S12). CC CL1 comprised of a total of 73 accessions and had more admixed individuals (48) than pure individuals (25) followed by CC CL4 consisting of 70 accessions (42 admixed and 28 pure individuals (Supplemental Table S12). The clusters CC CL2, 5 and 7 had approximately equal number of pure and admixed accessions while CC CL3 and CC CL6 had higher number of pure individuals in comparison to admixed accessions. Admixture assessment of varietal group of CC3 showed that Intermediate group had more admixtures (47) than pure (14) individuals followed by japonica with 12 admixed and 11 pure accessions. The indica group had 144 admixed genotypes (49\%) out of 295 accessions while Tropical japonica had 13 admixed accessions out of a total of 27 individuals. Analysis of regional gene pools of rice accessions revealed that South East Asia (63 admixed and 60 pure accessions), China (54 admixed and 47 pure accession), America (23 admixed and 15 pure accessions), Europe (13 admixed and 6 pure accessions) and Oceania (3 admixed and 2 pure accessions) pools had more admixed individuals than pure individuals present in CC3 (Supplemental Table S12). Detailed distribution of admixed and pure accessions from these different groups present in CC3 has been provided in Supplemental Table S12. Increased number of admixed individuals among populations derived using FastSTRUCTURE (CC CL1-CC CL7), varietal and geographical distribution/regional gene pool level confirms that $\mathrm{CC} 3$ comprised of more unrelated and admixed individuals in comparison to initial collection of rice and validates its suitability as an association panel. 


\section{Kinship analysis of mini-core $\mathrm{CC} 3$ individuals}

Kinship analysis between individuals of mini-core collection CC3 was performed to estimate coancestry among the accessions. Seventy percent of pairs of the CC3 accessions had a kinship value of less than zero while $25.4 \%$ accessions had kinship values ranging between $0-0.25$ percent (Fig.5). Around $4.5 \%$ of CC3 accessions showed kinship value in the range of $0.25-0.50 \%$, while only $0.1 \%$ of accessions had kinship value in the range of $0.5-0.75 \%$ (Fig. 5). Thus, the kinship value for most of the CC3 accessions exhibited absence or weak level of genetic relatedness among the individuals fulfilling the primary requirement of utilization of mini-core collection $\mathrm{CC} 3$ as an association panel.

\section{GWAS with mini-core collection CC3}

As the mini-core collection (CC3) showed low population structure and low kinship value, we proceeded to study its utilization for Genome Wide Association Studies (GWAS) in rice. GWAS analysis was performed on mini-core collection CC3 accessions (520) using 2081521 SNPs with MAF $>0.02$ and 18 yield-related traits of agronomic importance using the program GAPIT based on compressed mixed linear model (MLM). Association between markers and traits was considered to be significant at $P<1 \times 10^{-8}$, FDR adjusted $P$ value of $<0.05$ and correlation value $\left(\mathrm{R}^{2}\right)$ of $>=10 \%$. Six out of the 18 traits analysed namely Endosperm Type (ET), Grain Length (GL), Grain Width (GW), Panicle axis (PA), Secondary branching (SB) and Seed coat color (SCC) showed significant markertrait associations (Table 1). A total of 5924 SNPs were found to be significantly associated with the above mentioned six traits covering phenotypic variation from 10.4 to $61.6 \%$. Three SNPs were found to be significantly associated with the trait Grain Length (GL) in our analysis which was present on chromosome 3. The most significant SNP (G/T) associated with the trait GL on chromosome 3 was at the position 16733441 with FDR adjusted $P$ value of $1.4 \times 10^{-3}$ and explaining phenotypic variation of $32.2 \%$ (Fig. 6A). This was previously reported as known loci for GS3 (Fan et al., 2006). Another important grain trait, Grain Width $(\mathrm{GW})$ showed significant association with 64 SNPs specifically present on chromosome 5. The most significant SNP (C/G) associated with the trait GW on chromosome 5 at position 5371949 had FDR adjusted $P$ value of $2.8 \times 10^{-4}$ and explained phenotypic variation of $34.2 \%$ (Fig. 6B). This SNP was found to be associated with previously characterized locus qSW5 (Shomura et al., 2008). The trait Endosperm Type (ET) showed maximum number of significant associations with 3651 SNPs distributed on chromosomes 2, 4, 6, 8, 11 and 12 . The most significant SNP (G/T) was found to be present on chromosome 6 at position 6294468 with FDR adjusted $P$ value of $1.2 \times 10^{-8}$ and explaining $29 \%$ of phenotypic variation (Fig. 6C). The other significant SNP (T/G) associated with ET on chromosome 6 was found at position 1765761 with FDR adjusted $P$ value of $6.4 \times 10^{-8}$ and detecting a phenotypic variation of $25 \%$. This was also previously reported as known loci for Waxy gene by various authors (GAO, 2003; Tian et al., 2009; Huang et al., 
2010). The other significantly associated SNP were found to be present on chromosome number 2 at position $10277497(\mathrm{C} / \mathrm{T})$ with FDR adjusted $P$ value of $1.7 \times 10^{-7}$ and explaining phenotypic variation of $24 \%$ and on chromosome 8 at position 6048049 (G/A) with FDR adjusted $P$ value of $4.3 \times 10^{-7}$ and explaining phenotypic variation of $23.2 \%$ (Table 1). The trait Seed coat color (SCC) was found to be associated with 306 SNPs present on chromosome 7 in our analysis. The most significant SNP (T/C) associated with the trait SCC was present on chromosome 7 at position 6124457 with FDR adjusted $P$ value of $4.5 \times 10^{-8}$ and explaining a phenotypic variation of $61.6 \%$ (Fig. 6D). This SNP was found to be associated with $R c$ gene described in previous report as important locus for SCC (Sweeney et al., 2006). Another SNP significantly associated with the trait SCC (T/G) was present at position 6660825 on chromosome 7 with FDR adjusted $P$ value of $1.6 \times 10^{-6}$ and explaining phenotypic variation of $59.7 \%$. The trait Secondary branching (SB) was found to be associated with 1779 SNPs distributed on chromosomes 2, 4, 6, 7, 9 and 11. The most significant SNP (C/T) associated with the trait SB was present on chromosome 2 at position 5032535 with FDR adjusted $P$ value of $6.4 \times 10^{-7}$ and explaining a phenotypic variation of $32 \%$ (Fig. 6E). Significantly associated SNP with the trait SB were also present on chromosome 4 at position 2521459 (A/G) and 12427420 (G/A) with FDR adjusted $P$ value of $1.6 \times 10^{-4}$ each and explaining phenotypic variation of 23.5 and $23.4 \%$, respectively. The trait Panicle axis (PA) was significantly associated with 121 SNPs distributed on Chromosomes 2, 4, 6 and 10. The most significant SNP associated (A/C) with the trait PA was present on chromosome 4 at position 1075655 with FDR adjusted $P$ value of $3.7 \times 10^{-4}$ and explaining phenotypic variation of $24 \%$ (Fig. 6F). The SNPs that were significantly associated with the trait PA were also present on chromosomes 6 and 10 at positions $28676456(\mathrm{G} / \mathrm{A})$ and 14829875 (C/A) with FDR adjusted $P$ value of $3.7 \times 10^{-4}$ each and explaining phenotypic variation of 23.1 and

$23 \%$, respectively. Thus, identification of previously characterized QTLs for respective traits authenticates the utility and importance of mini-core CC3. The detailed distribution of all significantly associated 5924 SNPs, identified in the current analysis associated with the traits Endosperm Type (ET), Secondary branches (SB), Seed coat color (SCC), Panicle axis (PA), Grain Width (GW) and Grain Length (GL) and their chromosomal position has been provided in Supplemental Table S13.

\section{GWAS using original (3004 accessions) panel}

To further validate that the efficiency of designed mini-core collection CC3 (520 accessions) in terms of capturing the maximum possible marker-trait associations, we performed GWAS analyses with original collection of 3004 rice accessions for the above mentioned traits covering 500000 SNPs. The number of SNPs was reduced due to matrix limitations of the R program. In the case of original collection, only four [Endosperm Type (ET), Seed coat color (SCC), Grain Width (GW) and Grain Length (GL)] of the six traits showing significant marker-trait association in CC3 showed significant association. However, two traits namely 100 grain weight (HGW) and panicle threshability (PT) 
showed association in the case of original collection. These were missing in the analyses of CC3 minicore (Supplemental Table S14). Overall, 1790 SNPs were found to be significantly associated with different traits covering phenotypic variation ranging from 5.6-51.4\%. Grain Length (GL) was found to be associated with 325 SNPs present on chromosome 3 and 5. The most significant SNP (G/T) associated with the trait Grain Length (GL) present on chromosome 3 was at position 16733441 with FDR adjusted $P$ value of $3.4 \times 10^{-43}$ and explaining a phenotypic variation of $43.5 \%$ (Supplemental Figure S1A). Other SNP associated significantly with GL was found on chromosome 5 (G/A) at position 5361894 with FDR adjusted $P$ value of $1.03 \times 10^{-9}$ and detecting a phenotypic variation of 38.8\%. Grain Width (GW) could be significantly associated with 737 SNPs specifically present on chromosome 5. Consistent with the earlier studies, the most significant SNP on chromosome $5(\mathrm{C} / \mathrm{T})$ was found at position 5371686 with FDR adjusted $P$ value of $9.3 \times 10^{-34}$ and explaining $51.4 \%$ of phenotypic variation (Supplemental Figure S1B). The other significant SNP at chromosome 5 (T/C) associated with GW was at 28019687 with FDR adjusted $P$ value of $8.4 \times 10^{-6}$ and detecting a phenotypic variation of $48 \%$. The trait 100 grain weight (HGW) showed significant associations with 54 SNPs distributed on chromosomes 3 and 5. The most significant SNP was found to be present on chromosome $3(\mathrm{G} / \mathrm{T})$ at position 16733441 with FDR adjusted $P$ value of $7.9 \times 10^{-5}$ and explaining $35.2 \%$ of phenotypic variation. The other significant SNP at chromosome 5 (T/C) associated with HGW was at position 5375201 with FDR adjusted $P$ value of $7.9 \times 10^{-5}$ and detecting a phenotypic variation of 35.2\% (Supplemental Figure S1C). The Endosperm Type (ET) trait was found to be associated with 503 SNPs present on chromosome 6 . The most significant SNP (G/C) associated with the trait ET was present on chromosome 6 at position 1731808 with FDR adjusted $P$ value of $1.03 \mathrm{X}$ $10^{-29}$ and explaining a phenotypic variation of $20.2 \%$ (Supplemental Figure S1D). The other significant SNP at chromosome 6 (G/A) associated with ET was at position 6830286 with FDR adjusted $P$ value of $3.4 \times 10^{-8}$ and detecting a phenotypic variation of $15.6 \%$. The trait Seed coat color (SCC) showed significant associations with 170 SNPs distributed on chromosome 2 and 7. The most significant SNP was found to be present on chromosome 7 (G/A) at position 6133394 with FDR adjusted $P$ value of $6.6 \times 10^{-11}$ and explaining $7.2 \%$ of phenotypic variation (Supplemental Figure $\mathrm{S} 1 \mathrm{E})$. The other significant SNP on chromosome $7(\mathrm{G} / \mathrm{T})$ associated with SCC was at position 6417000 with FDR adjusted $P$ value of $1.7 \times 10^{-10}$ and detecting a phenotypic variation of $7.1 \%$. The other significantly associated SNP were found to be present on chromosome 7 (T/C) at position 6656052 with FDR adjusted $P$ value of $1.8 \times 10^{-8}$ and explaining phenotypic variation of $6.8 \%$ and on chromosome $2(\mathrm{~A} / \mathrm{G})$ at position 32431463 with FDR adjusted $P$ value of $3.7 \times 10^{-5}$ and explaining phenotypic variation of $5.6 \%$. The panicle threshability (PT) trait showed significant association with only 1 SNP present on chromosome $2(\mathrm{C} / \mathrm{T})$ at position 21739453 with FDR adjusted $P$ value of $6.8 \mathrm{X}$ $10^{-3}$ and explaining $16.4 \%$ of phenotypic variation (Supplemental Figure S1F). The detailed distribution of different SNPs associated with different traits such as Endosperm Type (ET), Seed coat 
color (SCC), Grain Width (GW), Grain Length (GL), 100 grain weight (HGW) and panicle threshability (PT) and their chromosomal positions for original collection of rice has been provided in Supplemental Table S14.

\section{GWAS based on regional and varietal grouping}

Association analysis was also performed on different set of accessions based on varietal and regional genepool. The aim is to identify novel marker-trait association specific to a particular group which might have not been captured in case of association analysis of developed mini-core collection (CC3) and original collection of 3004 accessions of rice. Among the regional genepools, strong association were observed for various traits like Endosperm Type (ET), Grain Length (GL), Grain Width (GW), Panicle axis (PA), Secondary branching (SB), Seed coat color (SCC), 100 grain weight (HGW) and panicle threshability (PT) as evident in case of $\mathrm{CC} 3$ and original collection. Interestingly, panicle shattering (PS) was found to have strong association among African genotypes which was absent in case of CC3 and original panel of rice accessions. The most significant SNP was found to be present on chromosome 7 at position 19458367 with FDR adjusted $P$ value of $2.9 \times 10^{-3}$ and explaining $29.4 \%$ of phenotypic variation (Supplemental Figure S2A). The other significant SNP at chromosome 3 associated with PS was at position 15574478 with FDR adjusted $P$ value of $3.3 \times 10^{-3}$ and detecting a phenotypic variation of $28 \%$.

In the case of varietal specific analysis, strong associations were again observed for numerous traits namely Endosperm Type (ET), Grain Length (GL), Grain Width (GW), Panicle axis (PA), Secondary branching (SB), Seed coat color (SCC), 100 grain weight (HGW) and panicle threshability (PT) as observed in CC3. Interestingly, here we identified one novel association for panicle shattering (PS) among the Temperate japonica group which was absent in the case of CC3 and the original panel of rice accessions. The most significant SNPs were found to be present on chromosomes 1 and chromosome 4 at positions 22646649 and 32213453 with FDR adjusted $P$ value of $1.6 \times 10^{-7}$ each, and explaining $61.5 \%$ of phenotypic variation each, respectively (Supplemental Figure S2B). The other significant SNP on chromosome 11 associated with PS was at position 4141451 with FDR adjusted $P$ value of $1.6 \times 10^{-7}$ and detecting a phenotypic variation of $54.7 \%$. The other significantly associated SNP was found to be present on chromosome 2 at position 4724352 with FDR adjusted $P$ value of $1.6 \times 10^{-7}$ and explaining phenotypic variation of $54.7 \%$ and on chromosome 9 at position 169498 with FDR adjusted $P$ value of $1.6 \times 10^{-7}$ and explaining phenotypic variation of $54.7 \%$. 


\section{Linkage disequilibrium and haplotype analysis}

To gain further insight into some of the less characterized marker-trait association such as panicle secondary branching found in the above analysis, the GWAS highlighted SNPs were studied for the LD pattern and haplotype block generation with their flanking nucleotides. In case of panicle secondary branching trait, 100 SNPs flanking the most associated SNP (SNP identity) were considered for the analysis and identification of the block containing the associated SNP. This block showed strong LD in a span of $2 \mathrm{~kb}$, containing 7 neighboring SNPs including the associated one (Fig. 7A). Haplotype analysis of this block revealed that PSB_H1 (ATCAGGT) haplotype had the highest frequency ( $\mathrm{f}=0.45$ ) among all the haplotypes. Distribution of these haplotypes between light and dense panicle secondary branching revealed all but $\mathrm{H} 4$ haplotype having significant association with light level branching (Fig. 7A). Contrastingly, H4 haplotype showed inclination towards dense branching trait. One recent study has shown the association of gene SHORT PANICLE 1 (SPI) and elevated haplotype diversity among japonica than indica group (Jang et al., 2018).

The grain length trait was also analyzed. LD analysis was performed for the identification of block containing the associated SNP. This block showed strong LD within a span of $1 \mathrm{~kb}$, containing 3 neighboring SNPs including the associated one (Fig. 7B). Haplotype analysis of this block revealed that GL_H1 (TTG) haplotype had the highest frequency ( $\mathrm{f}=0.631$ ) among all the haplotypes (GL_H2; TTT=0.315, GL_H3; TCG=0.034, GL_H4; CCG=0.013). Distribution of these haplotypes between long and short grain length revealed that GL_H2 in addition of being the second most frequent haplotype is also maximally associated with long rice grains, with average $9.33 \mathrm{~mm}$ grain length. Similarly, haplotype GL_H1 and GL_H3 identified with intermediate grain length i.e. showing mean value of 8.2 and $7.9 \mathrm{~mm}$, respectively (Fig. 7B). On the other hand, H4 haplotype showed inclination towards short grain trait, having the least mean grain length of $4.8 \mathrm{~mm}$. Above, haplotype observation was similar to one recent study dissecting the separate clustering of grain length haplotypes for varying size and different rice groups (Singh et al., 2017).

\section{GWAS correlation with expression data}

Significantly associated SNPs were analysed for their position on the genome and association with gene(s) using the annotation data from RGAP (Kawahara et al., 2013). Rice has an estimated linkage disequilibrium value of around $200 \mathrm{~Kb}$ due to its self-pollinating nature (McNally et al., 2009). Keeping this in view, a genomic stretch of $100 \mathrm{~Kb}$ up- and down-stream of the significantly associated SNPs was scanned to identify the genes. Expression level of genes/loci flanking the significantly associated SNPs was assessed in different tissues to be a probable candidate gene of function. The Grain Length (GL) trait which was found to be significantly associated with GS3 in earlier studies 
(Fan et al., 2006) was highlighted in our analysis. We found one SNP (16733441;G/T) on chromosome 3 significantly associated with GL. This SNP belonged to the gene LOC_Os03g29260 showing high expression in seed of 5 DAP, embryo at 25 DAP and in pre- and post-emerging inflorescence (RGAP; Table 1). The Grain Width (GW) trait was found to be significantly associated with qSW5 locus in earlier studies (Shomura et al., 2008). Here, we have identified identical SNP (C/G) at position 5371949 (chromosome 5; LOC_Os05g09500 and LOC_Os05g09550) showing strong association with Grain Width and higher expression in various tissues like seed of 5 DAP, embryo of 25 DAP and in pre- and post-emerging inflorescence (RGAP; Table 1). Significant markertrait associations were observed for two traits namely 100 grain weight (HGW) and panicle threshability (PT) in the original collection (Supplemental Table S14). Interestingly, same two SNPs namely SNP (5371949;C/G) on chromosome 5 and SNP $(16733441 ; \mathrm{G} / \mathrm{T})$ on chromosome 3, were identified to influence the HGW trait. These SNPs were identified to be associated with Grain Length (GL) and Grain Width (GW) traits in CC3 accessions. A major QTL governing the traits of gelatinization temperature and amylose content, which in-turn affects Endosperm Type (ET), has been identified on chromosome 6 in various studies (GAO, 2003; Tian et al., 2009; Huang et al., 2010). In our analysis of mini-core collection CC3, the SNP $(1765761 ; \mathrm{T} / \mathrm{G})$ on chromosome 6 was found to be associated with Endosperm Type (ET). The gene encompassing this SNP could be Waxy (LOC_Os06g04200) showing higher expression in the seed of 5-10 DAP and in the endosperm of 25 DAP (RGAP; Table 1). Two other SNPs, one on chromosome $6(6294468 ; \mathrm{G} / \mathrm{T})$ and other on chromosome 2 (C/G;7413964) showed association with the ET trait, and were flanked by genes LOC_Os06g11812 and LOC_Os02g13840, respectively. These genes are predominately expressed in seed. On the similar note, the Secondary Branching (SB) trait significantly associated with SNP (C/T) at position 5032535 (chromosome 2; LOC_Os02g09780), SNP (A/G) at position 2521459 (chromosome 4; LOC_Os04g05050) and SNP (G/A) at position 1827355 (chromosome 7; LOC_Os07g04190) showed higher expression level in various tissues such as seed at 5 DAP, shoots, pre- and post-emergence inflorescence (RGAP; Table 1). The Seed Coat Color (SCC) trait was found to be associated with $R c$ gene in earlier studies (Sweeney et al., 2006). In our analysis, we found SNP (6124457;T/C) on chromosome 7, flanked by LOC_Os07g11020, which showed association with $R c$ gene. We also found SNP (T/G) at position 6660825 (chromosome 7; LOC_Os07g11900) associated with the trait SCC trait showing high expression level in various tissues such as seed at 5 and 10 DAP, and endosperm at 25 DAP (RGAP; Table 1). The Panicle Axis (PA) trait was found to be significantly associated with SNP (A/C) at position 1075655 (chromosome 4; LOC_Os04g02820 and LOC_Os04g02900) and SNP (G/A) at position 28676456 (chromosome 6; LOC_Os06g47320, LOC_Os06g47340 and LOC_Os06g47350) and showed higher expression level in different tissues such as pre- and post-emergence inflorescence, shoot, pistil and embryo at 25 DAP (RGAP; Table 1). The Panicle Threshability (PT) trait was found to be significantly associated with SNP 
(21739453;C/T) at chromosome 2. The expression database suggests the exclusive induction of flanking gene LOC_Os02g36150 in post-inflorescence (RGAP), indicating its plausible involvement in the trait. Thus, we were able to validate the known loci for various traits identified in earlier studies showing the effectiveness of CC3 in GWAS of different traits. Along with that we have also identified new loci showing strong marker trait associations which can be studied further to understand the underlying genetic make-up of these traits.

\section{CONCLUSIONS}

Despite tremendous efforts, resolution of QTLs responsible for yield related traits and their causative genes have remained limited due to their complex nature. QTL mapping using a diverse panel and GWAS analysis has proven to be an effective tool to understand the genetic make-up. For GWAS analysis, estimation of underlying population structure of the panel under consideration is important which helps in avoiding spurious associations between phenotypes and genotypes (Pritchard and Rosenberg, 1999; Pritchard et al., 2000; Pritchard and Donnelly, 2001). Most of the earlier studies in rice have taken into consideration a particular population (Huang et al., 2010; Lu et al., 2015) which might have high level of structure and kinship for GWAS analysis resulting into spurious marker-trait associations. Here, this study for the first time utilized such large diversified (3004) rice germplasm, provided complete coverage of the global rice genepool. The mini-core was developed using program Core Hunter 3, based on 2 million genome-wide single nucleotide polymorphisms (SNPs), diverse 18 phenotypes and 89 country locations. The mini-core accounted for $17.3 \%$ of the original collection, and captured maximum of the SNP polymorphism, all phenotype and geographical regions. In addition, this panel showed low population structure and low or no kinship among the individuals of our panel, avoiding spurious marker-trait associations. Further, increase in number of admixed individuals in different clusters of structure analysis of CC3 showed that the panel was unstructured and diverse in nature, serving the purpose of using a mini-core for association analysis. Mini-core was validated using Shannon-Diversity Index.

On the utility front, the GWAS analyses with the designed mini-core panel found various novel marker-trait associations along with the validation of some of the earlier reported associations. More broadly, this analysis also provided a comparison between the mini-core $\mathrm{CC} 3$ and original collection and we were able to show that CC3 captured the associations prevalent in original collection and happens to be a representative subset. We found 5924 SNPs significantly associated with 6 different traits covering a phenotypic range of $10.4-61.6 \%$ in case of mini-core CC3, whereas identified 1790 SNPs significantly associated with 6 traits covering a phenotypic range of 4.9-51.4\% for the original collection. Association analysis performed on different set of accessions based on varietal and 
regional genepool led to the identification of novel marker-trait associations which were not captured in CC3 and original collection of rice for the trait panicle shattering. This suggests that one marker can be very effective in one set of accessions but not in the other set. The accessions can be linked to each other by the geographical region or the other context.

Expression analysis highlighted the associated SNPs flanking gene(s), which possibly be involved in respective agronomic trait development. Overall, we identified at least one gene showing expression abundance at expected development stage for all the six associated agronomic traits. This study also performed haplotype analysis for two specific traits; panicle secondary branching and grain length. The secondary branching was dealt as it was not so well studied whereas grain length haplotype analysis was performed to reproduce the previous studies within mini-core. In conclusion, we were able to generate and validate one mini-core as robust, diversified, non-redundant and manageable association panel, efficiently mirroring the large and diverse collection of 3004 rice accessions. We suggest this relatively small subset can be effectively utilized for efficient agronomic traits evaluation, which in-turn be useful for marker assisted breeding program for rice crop improvement.

\section{MATERIALS AND METHODS}

\section{Genotypic and phenotypic data of rice germplasm collection}

The present study utilized SNP data of 3004 rice accession (hereafter named as original collection) and the phenotypic data for 18 yield related traits i.e. days to $80 \%$ heading (DEH), 100 grain weight (HGW), endosperm type (ET), days to first flower (DFF), grain length (GL), grain width (GW), leaf senescence (LS), panicle axis (PA), panicle length (PL), panicle shattering (PS), panicle threshability (PT), secondary branches (SB), seed coat color (SCC), seedling height (SH), spikelet fertility (SF), culm length (CL), culm number (CN) and culm diameter (CD) of agronomic value for the development of mini-core collections and association analysis. The data of 3000 rice genome project (3K RGP) for polymorphic SNPs (18.9 million) along with phenotypic data were retrieved from SNPseek database (http://snp-seek.irri.org) (Alexandrov et al., 2015; Mansueto et al., 2017). In addition, we performed whole genome sequencing of 4 Indian accessions (LGR, PB-1121, Sonasal and Bindli) at a depth of $45 \mathrm{X}$ and collected phenotypic data for the above mentioned traits for the growing seasons 2016 and 2017. The accessions of initial collection of rice belonged to 89 countries representing all the regional pools and varieties of rice grown throughout the world. 


\section{Isolation of genomic DNA, genome sequencing and SNP calling}

All the four indian rice accessions [long grain: LGR (LG) and PB 1121 (PB); short grain: Sonasal (SN) and Bindli (BN)] were grown in the season of 2016 in NIPGR field. Rice seedling of 10 days old were used for the isolation of genomic DNA using Sigma GenEluteTM Plant gDNA kit. Integrity of genomic DNA was analyzed using Bioanalyzer 2100 (Agilent Technologies, Singapore). Samples for sequencing were prepared by Illumina TruSeq DNA sample preparation kit (Illumina, USA). Sequencing was performed with 90 bases pair-end chemistry on Illumina HiSeq 2000.

Subsequent standard bioinformatics analysis such as, raw reads quality check and removal of low quality bases (< Q30 Phred score) was done. The filtered reads were then mapped over the rice reference genome Nipponbare (IRGSP-1.0 pseudomolecule/MSU7) using BWA program with -q20 setting. Picard program was employed to get rid of duplicate reads.

Variant calling such single nucleotide polymorphisms (SNPs and InDels) were called via Genome Analysis TKLite-2.3-9 toolkit Unified Genotyper (GATK) (McKenna et al., 2010). The SNPs and InDels with a polymorphism call rate of $<90 \%$ were eliminated. After calling, in order to eliminate the low quality variants, a stringent of read depth $\geq 10$ and quality score $\geq 30$ was adopted to filter total variants and only good quality variants were considered for further analysis. Further, all the SNPs, those are consecutive and adjacent to InDels, were also eliminated. Plant Ensembl database was used to gene model for annotation. All the identified SNPs and InDels were annotated using customized VariMAT (SciGenome, India).

\section{Development of mini-core collections}

The program Core Hunter 3 (De Beukelaer et al., 2018) was used for development of independent mini-core collections using phenotypic and genotypic data. A cut off value of $10 \%$ of the initial collection was used to design the mini-core collections with default parameters. The mini-cores were also assessed for coverage of entire range for all the quantitative traits with reference to the initial collection. The diversity captured within the mini-core collections with respect to the initial collection was assessed using different evaluation indices such as Shannon's diversity index (I), Nei's gene diversity $(H)$, mean difference percentage (MD\%), variance difference percentage (VD\%), variable rate of coefficient of variance (VR\%) and coincidence rate of range (CR\%; Hu et al., 2000). Pearson correlation coefficient ( $\mathrm{r}$ ) was used to determine correlation between different quantitative traits using PAST version 3.10 (Hammer et al., 2001).

\section{Phylogenetic and population structure analysis}

The SNP data was utilized to construct distance-based dendrogram through Maximum-likelihood method using SNP Phylo program (Lee et al., 2014). Principal Coordinate Analysis (PCoA) was 
performed to estimate the overall relationship between accessions. Bayesian analysis of the population structure was performed using fastSTRUCTURE program (Raj et al., 2014) which estimated the optimal $\mathrm{K}$ value for the dataset. Pair-wise kinship coefficient was estimated using the program SPAGeDi (Hardy and Vekemans, 2002). To estimate the proportion of ancestral contribution for each accession admixture model was followed. The analysis was performed independent of the geographical and varietal origin of accessions. Accessions with $\mathrm{Q}$ value (membership proportion) >= $80 \%$ were considered as pure and designated into a particular cluster while accessions with $\mathrm{Q}$ value < $80 \%$ were considered as admixtures.

\section{Genome-wide association analysis}

All the Genome-wide association analysis were performed utilizing the program GAPIT (Lipka et al., 2012) based on compressed mixed linear model (MLM) for 18 rice agronomic traits. In case of original panel and developed mini-core, 2081521 and 500000 SNPs makers were utilized, respectively. Selected SNPs marker have minor allele frequency $[\mathrm{MAF}]>$ than 0.02 .

\section{Analysis of expression data}

Expression levels of all ORF's flanking $100 \mathrm{~Kb}$ up- and down-stream of significantly associated SNPs were assessed in different tissues within RGAP MSUv7 expression database (Kawahara et al., 2013). Further, gene loci showing preferential expression within plant organ responsible for trait development was notified as the plausible causative gene(s) associated studied trait.

\section{LD and Haplotype analyses}

Haplotypes were generated from the genotyped data. The linkage disequilibrium (LD) and haplotype analysis were performed using Haploview 4.2 (Barrett et al., 2005) with default parameters $(\mathrm{MAF}<0.001)$, HWE test $(<0.001)$ and percent genotypes test (cut of value $=75 \%)$. Four gamete rule method was employed to identify the more refined genomic block containing the associated SNP.

\section{ACKNOWLEDGMENTS}

This work was financially supported by the grants BT/AB/NIPGR/SEED BIOLOGY/2012 and for Sub-DIC facility from Department of Biotechnology (DBT), Government of India, to JKT. A.K acknowledges University Grant Commission and NIPGR for the Fellowships. S.K acknowledges NPDF (DST) and Short-Term Research Fellowship from NIPGR. The authors are thankful to DBTeLibrary Consortium (DeLCON) for providing access to literature. 


\section{$\underline{\text { Tables }}$}

Table 1: Association analysis of 520 accessions of mini-core collection CC3.

\begin{tabular}{|c|c|c|c|c|c|c|c|c|c|c|}
\hline Trait & Chr & Position & $\begin{array}{l}\text { Major } \\
\text { allele }\end{array}$ & $\begin{array}{l}\text { Minor } \\
\text { allele }\end{array}$ & $\begin{array}{l}\text { Minor allele } \\
\text { frequency }\end{array}$ & $\begin{array}{l}\text { Nipp. } \\
\text { allele }\end{array}$ & $\begin{array}{l}\mathrm{CC3} \\
\text { allele }\end{array}$ & $\begin{array}{c}\text { P-value } \\
\text { FDR } \\
\text { adjusted }\end{array}$ & $\begin{array}{c}\mathbf{R}^{2} \\
\text { value } \\
(\%)\end{array}$ & $\begin{array}{c}\text { Known } \\
\text { loci }\end{array}$ \\
\hline Grain length & 3 & 16733441 & G & $\mathrm{T}$ & 0.36 & G & $\mathrm{T}$ & $1.4 \times 10^{-3}$ & 32.3 & GS3 \\
\hline Grain width & 5 & 5371949 & $\mathrm{C}$ & G & 0.46 & $\mathrm{C}$ & G & $2.8 \times 10^{-4}$ & 34.2 & $q S W 5$ \\
\hline Endosperm type & 6 & 6294468 & G & $\mathrm{T}$ & 0.07 & G & $\mathrm{T}$ & $1.2 \times 10^{-8}$ & 29 & \\
\hline Endosperm type & 6 & 1765761 & $\mathrm{~T}$ & G & 0.13 & $\mathrm{~T}$ & G & $6.4 \times 10^{-8}$ & 25 & Waxy \\
\hline Endosperm type & 2 & 10277497 & $\mathrm{C}$ & $\mathrm{T}$ & 0.026 & $\mathrm{C}$ & $\mathrm{T}$ & $1.7 \times 10^{-7}$ & 24 & \\
\hline Endosperm type & 8 & 6048049 & G & A & 0.035 & G & A & $4.3 \times 10^{-7}$ & 23.2 & \\
\hline Seed coat color & 7 & 6124457 & $\mathrm{~T}$ & $\mathrm{C}$ & 0.456 & $\mathrm{~T}$ & $\mathrm{C}$ & $4.5 \times 10^{-8}$ & 61.6 & $R c$ \\
\hline Seed coat color & 7 & 6660825 & $\mathrm{~T}$ & G & 0.454 & $\mathrm{~T}$ & G & $1.6 \times 10^{-6}$ & 59.7 & \\
\hline Secondary branching & 2 & 5032535 & $\mathrm{C}$ & $\mathrm{T}$ & 0.013 & $\mathrm{C}$ & $\mathrm{T}$ & $6.4 \times 10^{-7}$ & 32 & \\
\hline Secondary branching & 4 & 2521459 & A & G & 0.052 & A & G & $1.6 \times 10^{-4}$ & 23.5 & \\
\hline Secondary branching & 4 & 12427420 & G & A & 0.208 & G & A & $1.6 \times 10^{-4}$ & 23.4 & \\
\hline Panicle axis & 4 & 1075655 & A & $\mathrm{C}$ & 0.013 & A & $\mathrm{C}$ & $3.7 \times 10^{-4}$ & 24 & \\
\hline Panicle axis & 6 & 28676456 & G & A & 0.0078 & G & A & $3.7 \times 10^{-4}$ & 23.1 & \\
\hline Panicle axis & 10 & 14829875 & $\mathrm{C}$ & A & 0.0078 & $\mathrm{C}$ & A & $3.7 \times 10^{-4}$ & 23 & \\
\hline
\end{tabular}

Nipp; Nipponbare, Chr; Chromosome 


\section{FIGURE LEGENDS}

Figure 1: Venn diagram showing distribution of accessions in different mini-core collections developed in the present analysis. $\mathrm{CC} 1$ represents mini-core designed using phenotypic data, CC2 represents mini-core designed using SNP data, CC3 represents merged mini-core collection.

Figure 2: Phylogenetic representation among 3004 rice accessions based on polymorphic SNP markers. (A) Maximum-likelihood dendrogram illustrating the genetic relationship among. Two clusters designated as $\mathrm{Cl}$ I-II with further sub-clustering are shown. (B) Principal Component Analysis of 3004 accessions of original collection for principal component axes 1 and 2. Distribution of accessions in different quadrants (I-IV) is shown. Varietal group color codes are provided in the figure.

Figure 3: Phylogenetic representation showing distribution of CC3 accessions. (A) Distribution of CC3 accessions (520) in different clusters of maximum-likelihood dendrogram of original collection of rice (3004 accessions). CC3 accessions are shown with red dots. (B) Principal Component Analysis of 520 accessions of mini-core collection CC3 for principal component axes 1 and 2. Distribution of accessions in different quadrants (I-IV) is shown. Varietal group color codes are provided in the figure.

Figure 4: Population structure of original (3004) and mini-core (520) rice accessions at $\mathrm{K}=7$, derived using the model based Bayesian algorithm. (A) Population structure of original (3004), each sub-population is represented by a different color code named as FSTR CL1- FSTR CL7.(B) Population structure of 520 rice accessions, each sub-population is represented by a different color code named as CC CL1- CC CL7. Each vertical bar represents a single rice accession. Admixed individuals have bars composed of multiple colors.

Figure 5: Kinship analysis between 520 accessions of mini-core CC3. (A) Histogram showing kinship status among mini-core accessions. (B) Kinship matrix for mini-core showing relatedness among accessions.

Figure 6: Genome-wide mapping for associations among CC3 (520) accessions with various yield-related domesticated traits. (A) Manhattan and QQ plots of compressed MLM for grain length. Negative $\log _{10^{-}}$ transformed $P$ values (y axis) values from the compressed mixed linear model are plotted against position (x axis) on different chromosomes. (B) Manhattan and QQ plots of compressed MLM for grain width, as plotted in A. (C) Manhattan and QQ plots of compressed MLM for endosperm type, as plotted in A. (D) Manhattan and QQ plots of compressed MLM for seed coat color, as plotted in A. (E) Manhattan and QQ plots of compressed MLM for secondary branching, as plotted in A. (F) Manhattan and QQ plots of compressed MLM for panicle axis, as plotted in A. Green line in each figure represents the genome-wide cut-off for significant association.

Figure 7: Linkage disequilibrium (LD) and haplotype analysis. (A) Depiction of strong LD and haplotype block containing the GWAS identified SNP for panicle secondary branching (PSB). The table shows the distribution of various haplotype for panicle secondary branching trait among core population. (B) Depiction of strong LD and haplotype block containing the GWAS identified SNP for grain length (GL). The table shows the distribution of various haplotype for panicle secondary branching trait among core population. GWAS identified SNP is highlight in blue. SNP id in bold format depicts Tag SNP. Red blocks, D' (normalized linkage disequilibrium measure or $\mathrm{D}) \leq 1.0$, with logarithm of odds (LOD) score $\geq 2.0$; white blocks, $\mathrm{D}^{\prime}<1.0$ with LOD $<2.0$; blue blocks, $\mathrm{D}^{\prime}=1.0$ with LOD $<2.0$. Numbers in blocks denote $\mathrm{D}^{\prime}$ values. The genomic organization is described above the LD plot. LOD was defined as $\log 10(\mathrm{~L} 1 / \mathrm{L} 0)$, where $\mathrm{L} 1=$ likelihood of the data under linkage disequilibrium, and LO = likelihood of the data under linkage equilibrium. 


\section{REFERENCES}

Alexandrov N, Tai S, Wang W, Mansueto L, Palis K, Fuentes RR, Ulat VJ, Chebotarov D, Zhang G, Li Z, et al (2015) SNP-Seek database of SNPs derived from 3000 rice genomes. Nucleic Acids Res 43: D1023-D1027

Ambreen H, Kumar S, Kumar A, Agarwal M, Jagannath A, Goel S (2018) Association Mapping for Important Agronomic Traits in Safflower (Carthamus tinctorius L.) Core Collection Using Microsatellite Markers. Front Plant Sci 9: 402

El Bakkali A, Haouane H, Moukhli A, Costes E, Van Damme P, Khadari B (2013) Construction of Core Collections Suitable for Association Mapping to Optimize Use of Mediterranean Olive (Olea europaea L.) Genetic Resources. PLoS One 8: e61265

Barrett JC, Fry B, Maller J, Daly MJ (2005) Haploview: analysis and visualization of LD and haplotype maps. Bioinformatics 21: 263-265

De Beukelaer H, Davenport GF, Fack V (2018) Core Hunter 3: flexible core subset selection. BMC Bioinformatics 19: 203

Breseghello F, Sorrells ME (2006) Association Analysis as a Strategy for Improvement of Quantitative Traits in Plants. Crop Sci 46: 1323

Brown AHD (2011) Core collections: a practical approach to genetic resources management. Genome 31: $818-824$

Brown AHD, Spilllane C (1999) Implementing core collections - principles, procedures, progress, problems and promise BT - Core Collections for Today and Tomorrow. Core Collect. Today Tomorrow. pp 7-17

Edgerton MD (2009) Increasing Crop Productivity to Meet Global Needs for Feed, Food, and Fuel. PLANT Physiol 149: 7-13

Famoso AN, Zhao K, Clark RT, Tung C-W, Wright MH, Bustamante C, Kochian L V., McCouch SR (2011) Genetic Architecture of Aluminum Tolerance in Rice (Oryza sativa) Determined through Genome-Wide Association Analysis and QTL Mapping. PLoS Genet 7: e1002221

Fan C, Xing Y, Mao H, Lu T, Han B, Xu C, Li X, Zhang Q (2006) GS3, a major QTL for grain length and weight and minor QTL for grain width and thickness in rice, encodes a putative transmembrane protein. Theor Appl Genet 112: 1164-1171

Fuentes RR, Chebotarov D, Duitama J, Smith S, De la Hoz JF, Mohiyuddin M, Wing RA, McNally KL, Tatarinova T, Grigoriev A, et al (2019) Structural variants in 3000 rice genomes. Genome Res 29: $870-880$

GAO Z (2003) Map-based cloning of the ALK gene, which controls the gelatinization temperature of rice. Sci China Ser C 46: 661

Gupta PK, Rustgi S, Kulwal PL (2005) Linkage disequilibrium and association studies in higher plants: Present status and future prospects. Plant Mol Biol 57: 461-485

Hammer Ø, Harper D, Ryan P (2001) Past: Paleontological statistics software package for education and data analysis. Paleontol. Electron.

Hardy OJ, Vekemans X (2002) spagedi: a versatile computer program to analyse spatial genetic structure at the individual or population levels. Mol Ecol Notes 2: 618-620

Huang X, Wei X, Sang T, Zhao Q, Feng Q, Zhao Y, Li C, Zhu C, Lu T, Zhang Z, et al (2010) Genome-wide association studies of 14 agronomic traits in rice landraces. Nat Genet 42: 961-967 
Huang X, Zhao Y, Wei X, Li C, Wang A, Zhao Q, Li W, Guo Y, Deng L, Zhu C, et al (2012) Genome-wide association study of flowering time and grain yield traits in a worldwide collection of rice germplasm. Nat Genet 44: 32-39

Ingvarsson PK, Street NR (2011) Association genetics of complex traits in plants. New Phytol 189: 909922

Jang S, Lee Y, Lee G, Seo J, Lee D, Yu Y, Chin JH, Koh H-J (2018) Association between sequence variants in panicle development genes and the number of spikelets per panicle in rice. BMC Genet 19: 5

Kawahara Y, de la Bastide M, Hamilton JP, Kanamori H, McCombie WR, Ouyang S, Schwartz DC, Tanaka T, Wu J, Zhou S, et al (2013) Improvement of the Oryza sativa Nipponbare reference genome using next generation sequence and optical map data. Rice (N Y) 6: 4

Korte A, Vilhjálmsson BJ, Segura V, Platt A, Long Q, Nordborg M (2012) A mixed-model approach for genome-wide association studies of correlated traits in structured populations. Nat Genet 44: 1066-1071

Kump KL, Bradbury PJ, Wisser RJ, Buckler ES, Belcher AR, Oropeza-Rosas MA, Zwonitzer JC, Kresovich S, McMullen MD, Ware D, et al (2011) Genome-wide association study of quantitative resistance to southern leaf blight in the maize nested association mapping population. Nat Genet $\mathbf{4 3}$ : $163-168$

Lee T-H, Guo H, Wang X, Kim C, Paterson AH (2014) SNPhylo: a pipeline to construct a phylogenetic tree from huge SNP data. BMC Genomics 15: 162

Li Z, Rutger J, Yu S, Xu W, Vijayakumar C, Ali J, Fu B, Xu J, Marghirang R, Domingo J, et al (2014) The 3,000 rice genomes project. Gigascience 3: 7

Lipka AE, Tian F, Wang Q, Peiffer J, Li M, Bradbury PJ, Gore MA, Buckler ES, Zhang Z (2012) GAPIT: genome association and prediction integrated tool. Bioinformatics 28: 2397-2399

Lu Q, Zhang M, Niu X, Wang S, Xu Q, Feng Y, Wang C, Deng H, Yuan X, Yu H, et al (2015) Genetic variation and association mapping for 12 agronomic traits in indica rice. BMC Genomics 16: 1067

Mansueto L, Fuentes RR, Borja FN, Detras J, Abrio-Santos JM, Chebotarov D, Sanciangco M, Palis K, Copetti D, Poliakov A, et al (2017) Rice SNP-seek database update: New SNPs, indels, and queries. Nucleic Acids Res 45: D1075-D1081

Mansueto L, Fuentes RR, Chebotarov D, Borja FN, Detras J, Abriol-Santos JM, Palis K, Poliakov A, Dubchak I, Solovyev V, et al (2016) SNP-Seek II: A resource for allele mining and analysis of big genomic data in Oryza sativa. Curr Plant Biol 7-8: 16-25

McCouch SR, Wright MH, Tung C-W, Maron LG, McNally KL, Fitzgerald M, Singh N, DeClerck G, Agosto-Perez F, Korniliev P, et al (2016) Open access resources for genome-wide association mapping in rice. Nat Commun 7: 10532

McKenna A, Hanna M, Banks E, Sivachenko A, Cibulskis K, Kernytsky A, Garimella K, Altshuler D, Gabriel S, Daly M, et al (2010) The Genome Analysis Toolkit: A MapReduce framework for analyzing next-generation DNA sequencing data. Genome Res 20: 1297-1303

McNally KL, Childs KL, Bohnert R, Davidson RM, Zhao K, Ulat VJ, Zeller G, Clark RM, Hoen DR, Bureau TE, et al (2009) Genomewide SNP variation reveals relationships among landraces and modern varieties of rice. Proc Natl Acad Sci 106: 12273-12278

Morrell PL, Buckler ES, Ross-Ibarra J (2012) Crop genomics: advances and applications. Nat Rev Genet 13: 85-96 
Nachimuthu VV, Muthurajan R, Duraialaguraja S, Sivakami R, Pandian BA, Ponniah G, Gunasekaran K, Swaminathan M, K K S, Sabariappan R (2015) Analysis of Population Structure and Genetic Diversity in Rice Germplasm Using SSR Markers: An Initiative Towards Association Mapping of Agronomic Traits in Oryza Sativa. Rice 8: 30

Perseguini JMKC, Silva GMB, Rosa JRBF, Gazaffi R, Marçal JF, Carbonell SAM, Chiorato AF, Zucchi MI, Garcia AAF, Benchimol-Reis LL (2015) Developing a common bean core collection suitable for association mapping studies. Genet Mol Biol 38: 67-78

Pritchard JK, Donnelly P (2001) Case-control studies of association in structured or admixed populations. Theor Popul Biol 60: 227-237

Pritchard JK, Rosenberg NA (1999) Use of Unlinked Genetic Markers to Detect Population Stratification in Association Studies. Am J Hum Genet 65: 220-228

Pritchard JK, Stephens M, Donnelly P (2000) Inference of population structure using multilocus genotype data. Genetics 155: 945-959

Raj A, Stephens M, Pritchard JK (2014) fastSTRUCTURE: Variational Inference of Population Structure in Large SNP Data Sets. Genetics 197: 573-589

Shomura A, Izawa T, Ebana K, Ebitani T, Kanegae H, Konishi S, Yano M (2008) Deletion in a gene associated with grain size increased yields during rice domestication. Nat Genet 40: 1023-1028

Singh N, Singh B, Rai V, Sidhu S, Singh AK, Singh NK (2017) Evolutionary Insights Based on SNP Haplotypes of Red Pericarp, Grain Size and Starch Synthase Genes in Wild and Cultivated Rice. Front Plant Sci 8: 972

Sweeney MT, Thomson MJ, Pfeil BE, McCouch S (2006) Caught red-handed: Rc encodes a basic helixloop-helix protein conditioning red pericarp in rice. Plant Cell 18: 283-294

Tian M, Tan G, Liu Y, Rong T, Huang Y (2009) Origin and evolution of Chinese waxy maize: Evidence from the Globulin-1 gene. Genet Resour Crop Evol 56: 247-255

Wang W, Mauleon R, Hu Z, Chebotarov D, Tai S, Wu Z, Li M, Zheng T, Fuentes RR, Zhang F, et al (2018) Genomic variation in 3,010 diverse accessions of Asian cultivated rice. Nature 557: 43-49

Yang X, Yan J, Shah T, Warburton ML, Li Q, Li L, Gao Y, Chai Y, Fu Z, Zhou Y, et al (2010) Genetic analysis and characterization of a new maize association mapping panel for quantitative trait loci dissection. Theor Appl Genet 121: 417-431

Yu J, Buckler ES (2006) Genetic association mapping and genome organization of maize. Curr Opin Biotechnol 17: 155-160

Zhang P, Liu X, Tong H, Lu Y, Li J (2014) Association Mapping for Important Agronomic Traits in Core Collection of Rice (Oryza sativa L.) with SSR Markers. PLoS One 9: e111508

Zhao K, Tung C-W, Eizenga GC, Wright MH, Ali ML, Price AH, Norton GJ, Islam MR, Reynolds A, Mezey J, et al (2011) Genome-wide association mapping reveals a rich genetic architecture of complex traits in Oryza sativa. Nat Commun 2: 467

Zhu C, Gore M, Buckler ES, Yu J (2008) Status and Prospects of Association Mapping in Plants. Plant Genome J 1: 5 


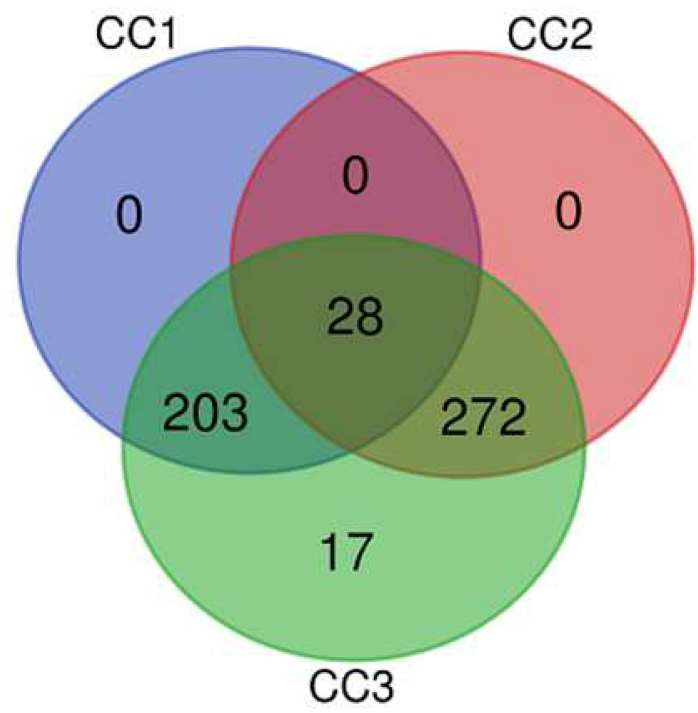

Figure 1: Venn diagram showing distribution of accessions in different mini-core collections developed in the present analysis. $\mathrm{CC} 1$ represents mini-core designed using phenotypic data, $\mathrm{CC} 2$ represents mini-core designed using SNP data, CC3 represents merged mini-core collection. 
A

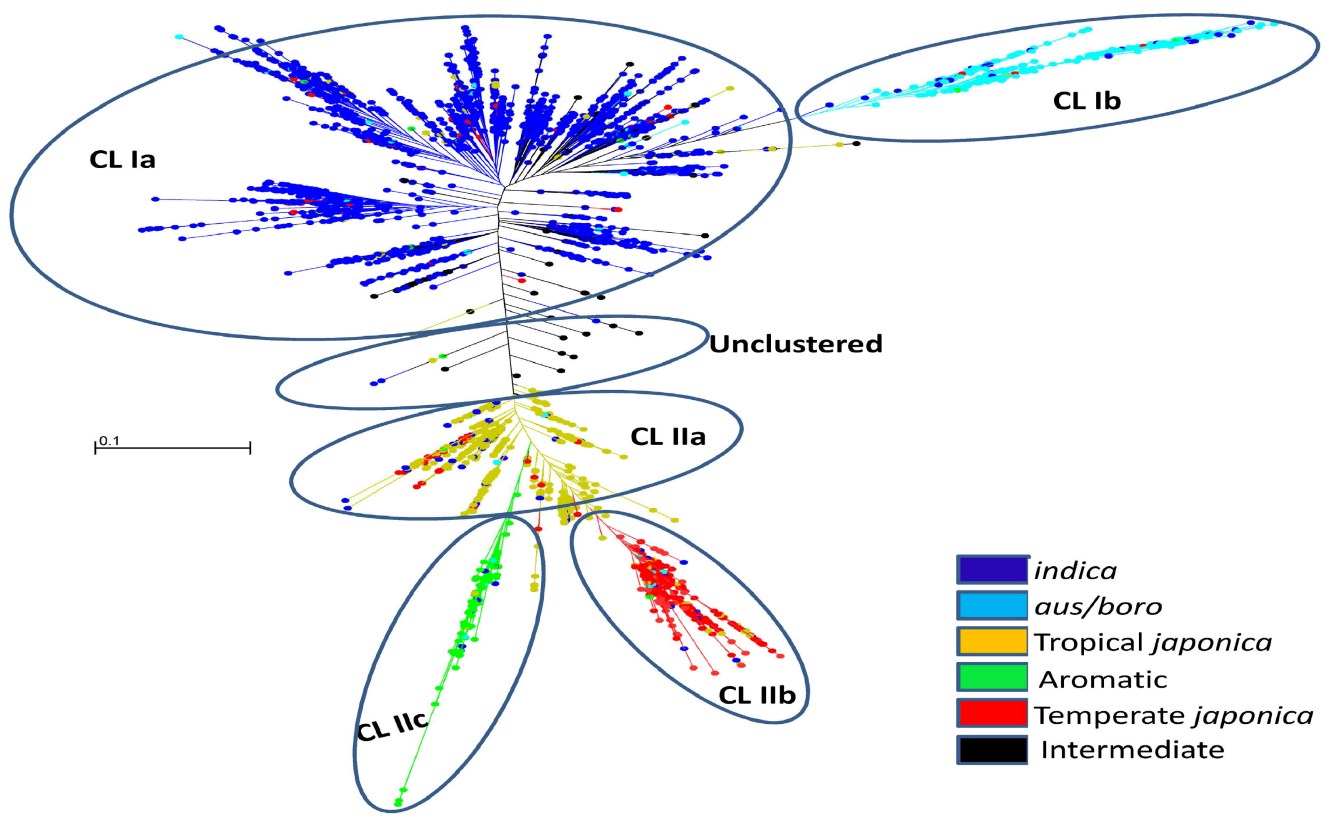

B

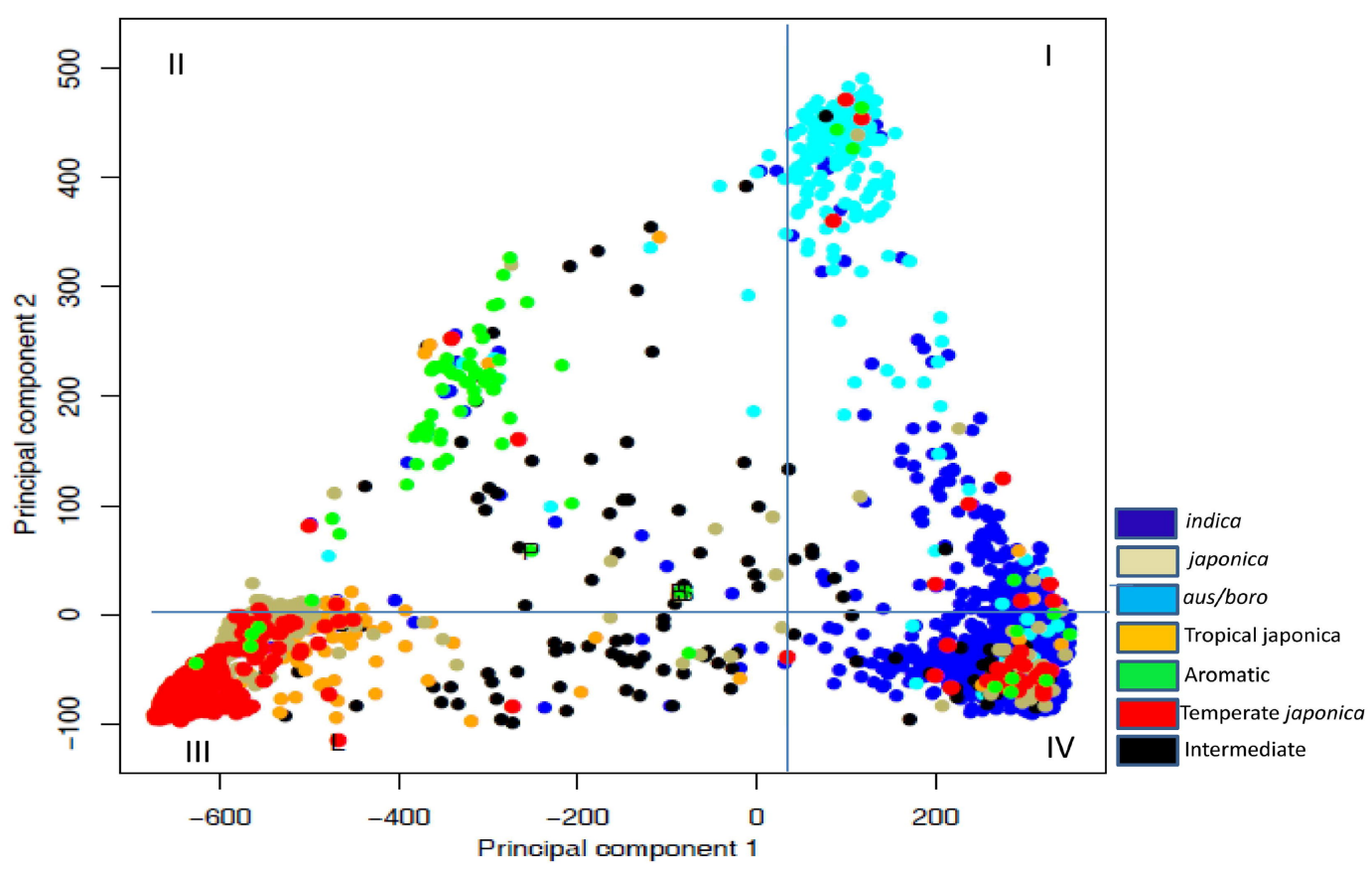

Figure 2: Phylogenetic representation among 3004 rice accessions based on polymorphic SNP markers. (A) Maximum-likelihood dendrogram illustrating the genetic relationship among. Two clusters designated as $\mathrm{Cl}$ I-II with further sub-clustering are shown. (B) Principal Component Analysis of 3004 accessions of original collection for principal component axes 1 and 2. Distribution of accessions in different quadrants (I-IV) is shown. Varietal group color codes are provided in the figure. 
A

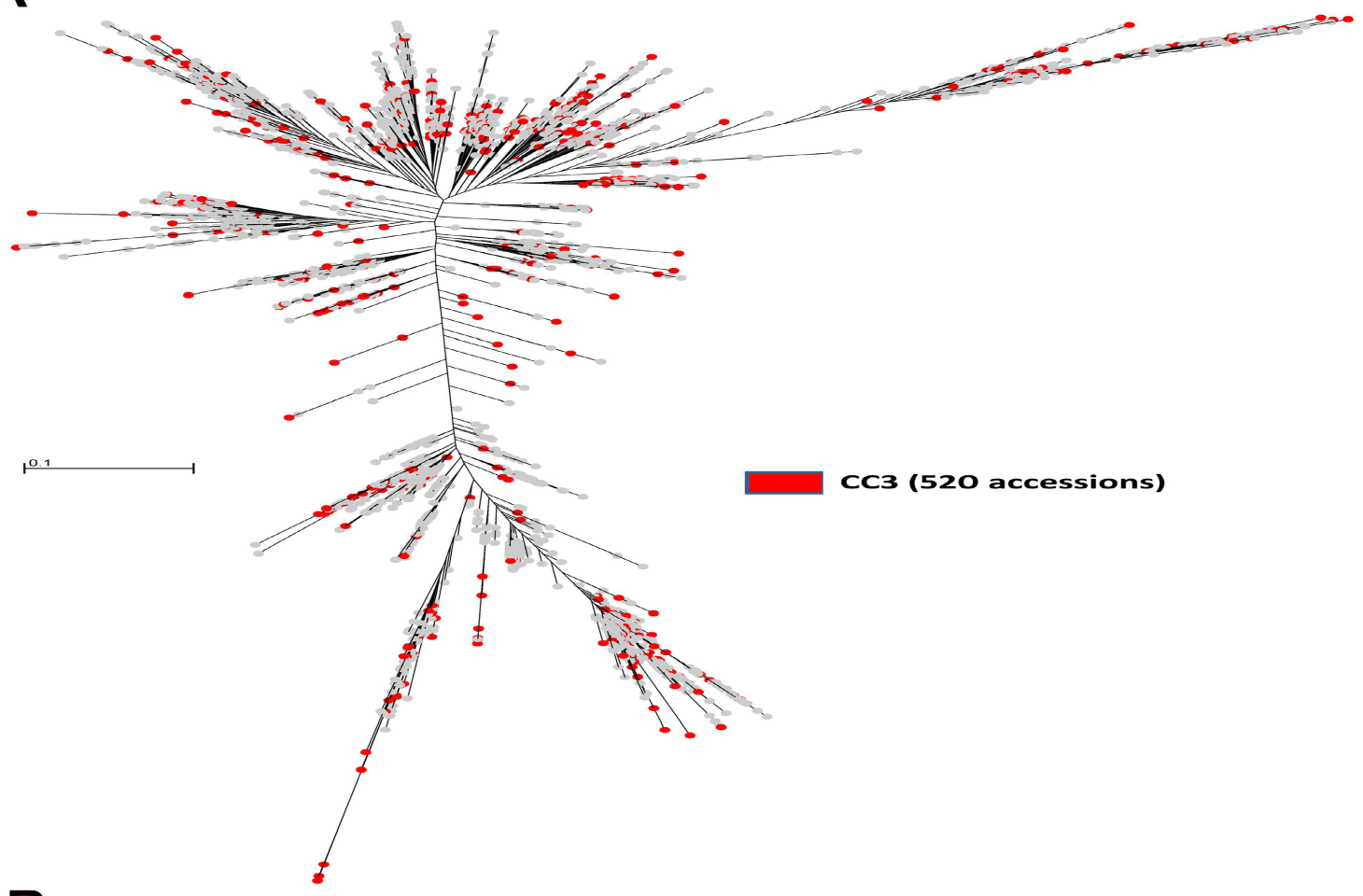

B

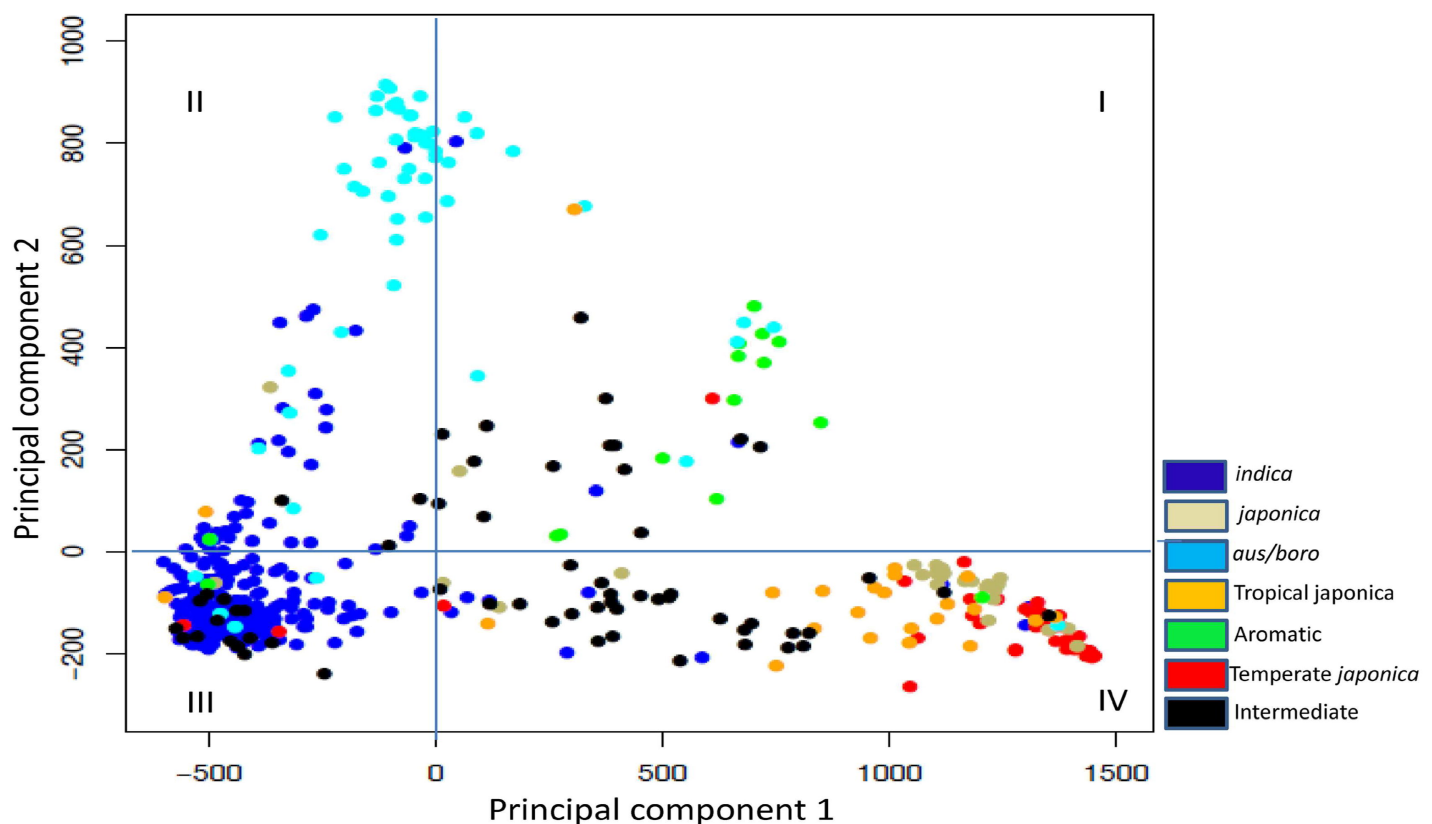

Figure 3: Phylogenetic representation showing distribution of CC3 accessions. (A) Distribution of CC3 accessions (520) in different clusters of maximum-likelihood dendrogram of original collection of rice (3004 accessions). CC3 accessions are shown with red dots. (B) Principal Component Analysis of 520 accessions of mini-core collection CC 3 for principal component axes 1 and 2. Distribution of accessions in different quadrants (I-IV) is shown. Varietal group color codes are provided in the figure. 

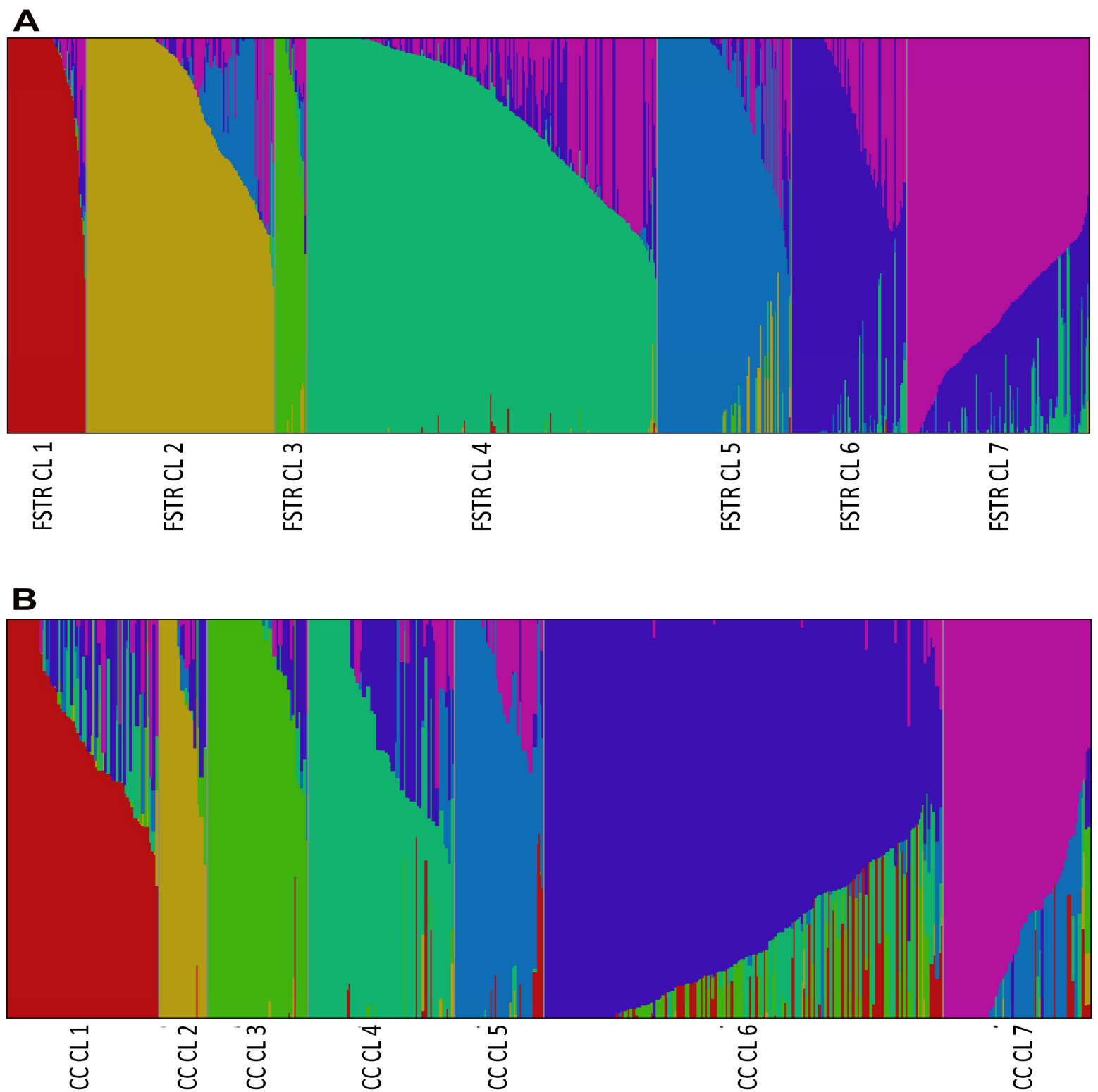

Figure 4: Population structure of original (3004) and mini-core (520) rice accessions at $K=7$, derived using the model based Bayesian algorithm. (A) Population structure of original (3004), each sub-population is represented by a different color code named as FSTR CL1- FSTR CL7.(B) Population structure of 520 rice accessions, each sub-population is represented by a different color code named as CC CL1- CC CL7. Each vertical bar represents a single rice accession. Admixed individuals have bars composed of multiple colors. 

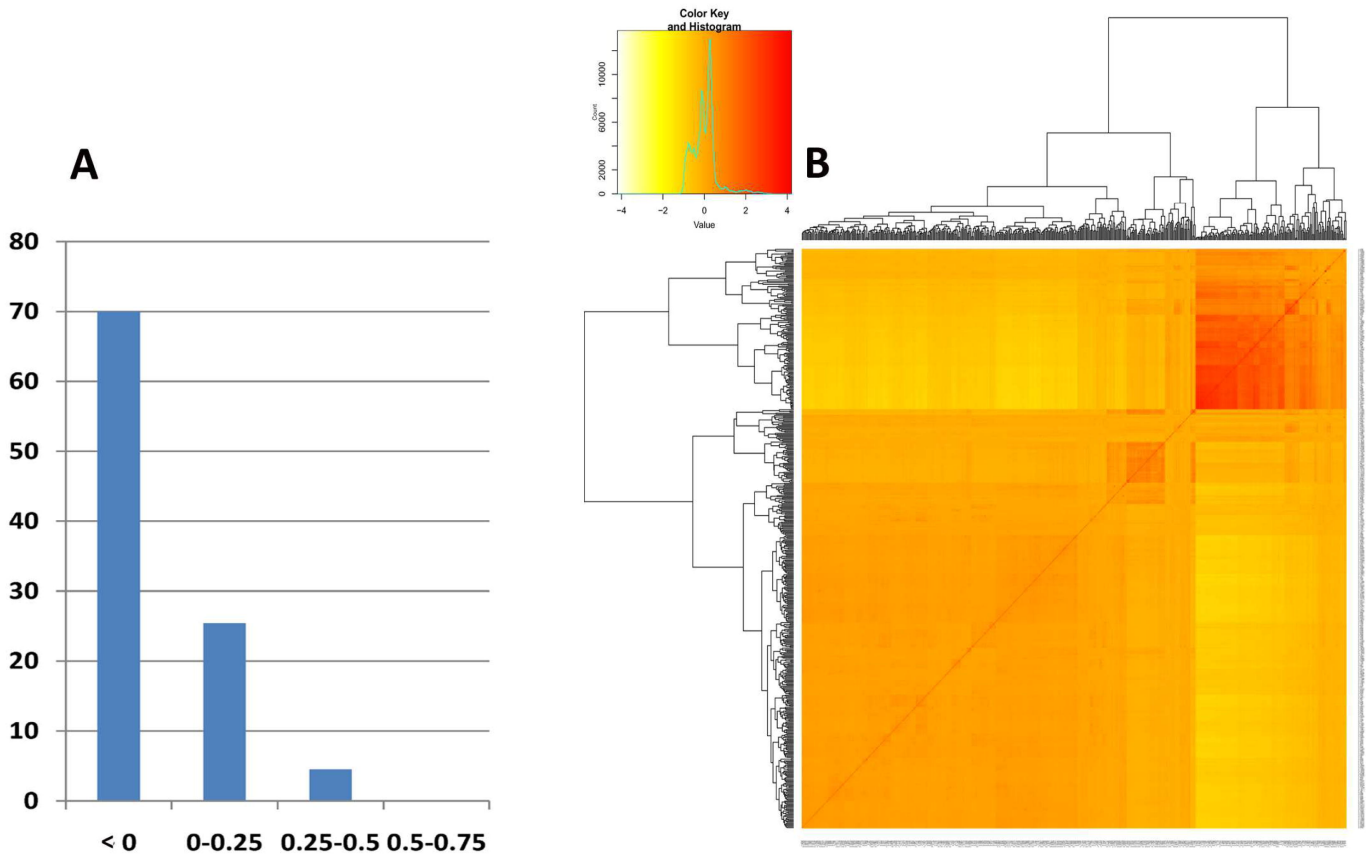

Figure 5: Kinship analysis between 520 accessions of mini-core CC3. (A) Histogram showing kinship status among mini-core accessions. (B) Kinship matrix for mini-core showing relatedness among accessions. 

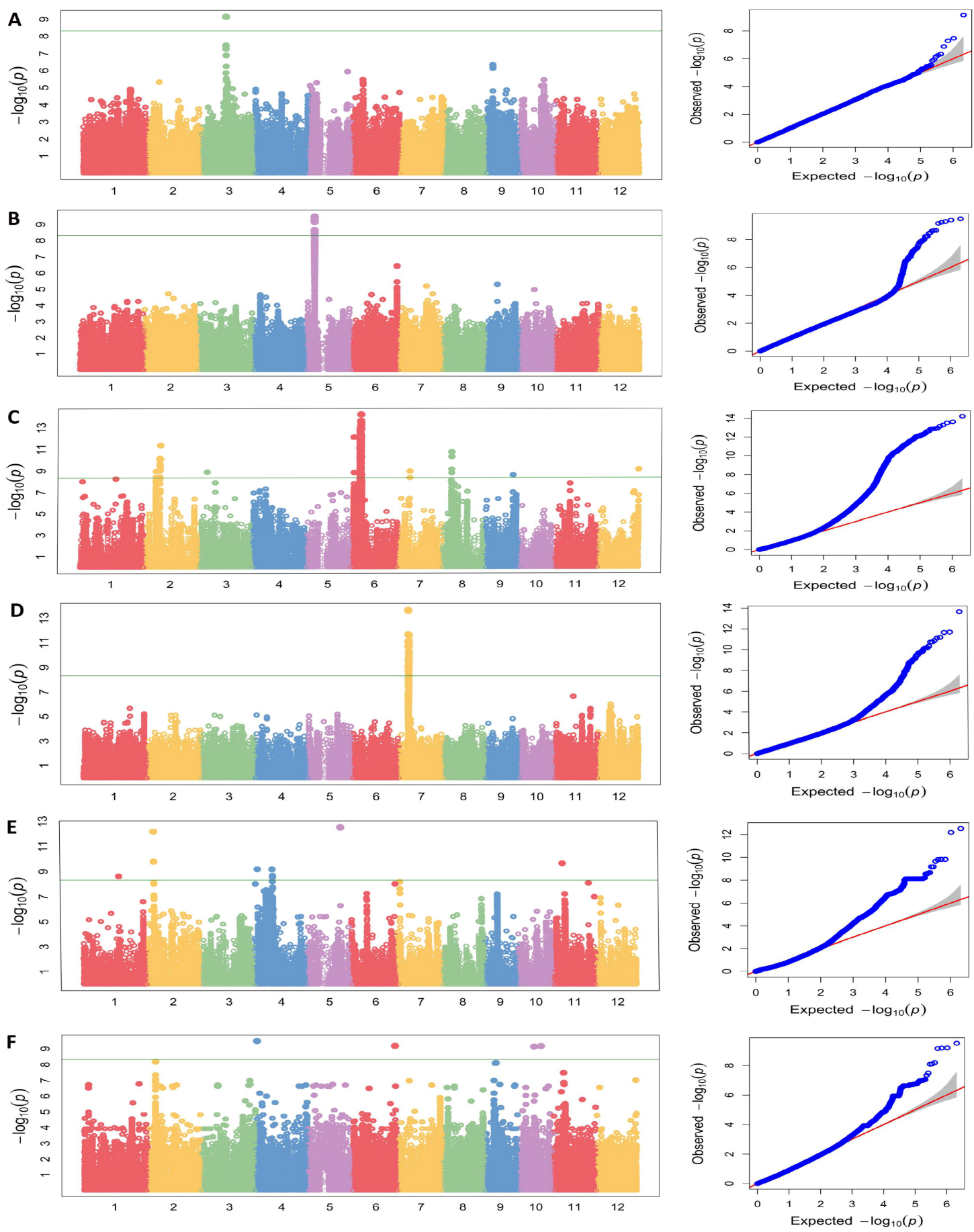

Figure 6: Genome-wide mapping for associations among CC3 (520) accessions with various yield-related domesticated traits. (A) Manhattan and QQ plots of compressed MLM for grain length. Negative $\log _{10}$-transformed $P$ values (y axis) values from the compressed mixed linear model are plotted against position (x axis) on different chromosomes. (B) Manhattan and QQ plots of compressed MLM for grain width, as plotted in A. (C) Manhattan and QQ plots of compressed MLM for endosperm type, as plotted in A. (D) Manhattan and QQ plots of compressed MLM for seed coat color, as plotted in A. (E) Manhattan and QQ plots of compressed MLM for secondary branching, as plotted in A. (F) Manhattan and QQ plots of compressed MLM for panicle axis, as plotted in A. Green line in each figure represents the genome-wide cut-off for significant association. 

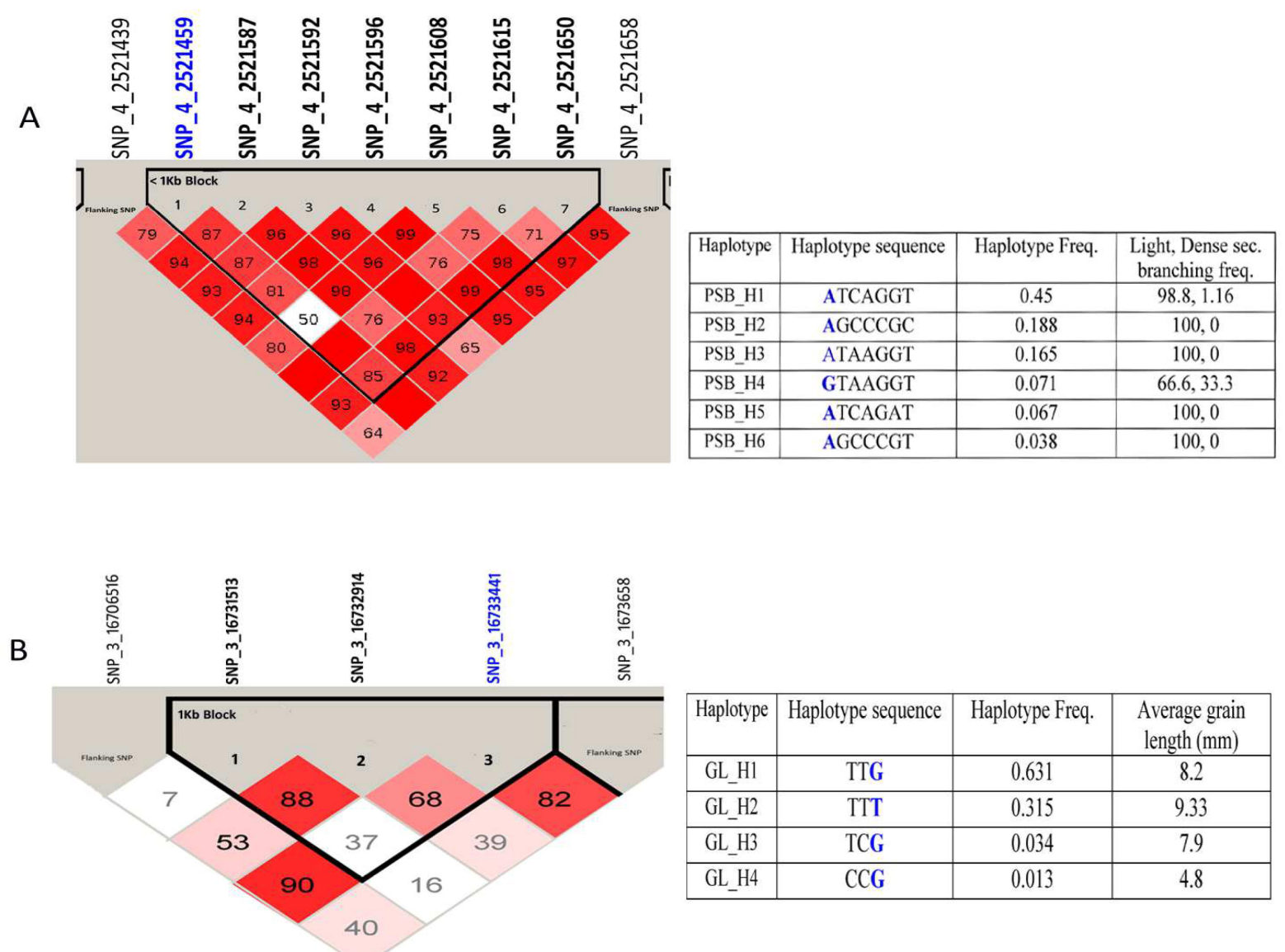

\begin{tabular}{|l|c|c|c|}
\hline Haplotype & Haplotype sequence & Haplotype Freq. & $\begin{array}{c}\text { Average grain } \\
\text { length }(\mathrm{mm})\end{array}$ \\
\hline GL_H1 & TTG & 0.631 & 8.2 \\
\hline GL_H2 & TTT & 0.315 & 9.33 \\
\hline GL_H3 & TCG & 0.034 & 7.9 \\
\hline GL_H4 & CCG & 0.013 & 4.8 \\
\hline
\end{tabular}

Figure 7: Linkage disequilibrium (LD) and haplotype analysis. (A) Depiction of strong LD and haplotype block containing the GWAS identified SNP for panicle secondary branching (PSB). The table shows the distribution of various haplotype for panicle secondary branching trait among core population. (B) Depiction of strong LD and haplotype block containing the GWAS identified SNP for grain length (GL). The table shows the distribution of various haplotype for panicle secondary branching trait among core population. GWAS identified SNP is highlight in blue. SNP id in bold format depicts Tag SNP. Red blocks, D' (normalized linkage disequilibrium measure or D) $\leq 1.0$, with logarithm of odds (LOD) score $\geq 2.0$; white blocks, $\mathrm{D}^{\prime}<1.0$ with LOD $<2.0$; blue blocks, $\mathrm{D}^{\prime}=1.0$ with $\mathrm{LOD}<2.0$. Numbers in blocks denote $\mathrm{D}^{\prime}$ values. The genomic organization is described above the LD plot. LOD was defined as $\log 10(\mathrm{~L} 1 / \mathrm{L} 0)$, where L1 = likelihood of the data under linkage disequilibrium, and L0 = likelihood of the data under linkage equilibrium. 